This manuscript has been submitted for publication in the Journal of Geophysical Research: Solid Earth. The article has not yet been peer reviewed and subsequent versions of this manuscript may be different. If accepted, the final version of this manuscript will be available via the 'Peerreviewed Publication DOI' link on the right-hand side of this webpage. Please feel free to contact the corresponding author, we welcome feedback

This article has been accepted for publication and $\bar{c}^{-1}$ nndergone full peer review but has not been through the copyediting, typesetting, pagination and proofreading process, which may lead to differences between this version and the Version of Record. Please cite this article as doi: 10.1029/2021JB023103.

This article is protected by copyright. All rights reserved. 


\title{
Parsimonious velocity inversion applied to the Los Angeles Basin, CA
}

\author{
Jack B. Muir ${ }^{1,2}$, Robert W. Clayton ${ }^{1}$, Victor C. Tsai ${ }^{3}$, and Quentin Brissaud ${ }^{4}$ \\ ${ }^{1}$ Seismological Laboratory, Division of Geological and Planetary Sciences, California Institute of \\ Technology, Pasadena, CA, USA \\ ${ }^{2}$ Research School of Earth Sciences, Australian National University, Acton, ACT, Australia \\ ${ }^{3}$ Department of Earth, Environmental and Planetary Sciences, Brown University, Providence, RI, USA \\ ${ }^{4}$ NORSAR, Oslo, Norway
}

\section{Key Points:}

- We generate a new velocity model of the northeastern Los Angeles Basin using data from the Community Seismic Network

- Using a level-set framework, we parsimoniously balance the existing Community Velocity Models with new data constraints

- The new model indicates a steeper and deeper basin underneath downtown Los Angeles, significantly amplifying 4-6 s Love waves

Corresponding author: Jack B. Muir, jmuir@caltech.edu 
Abstract

The proliferation of dense arrays promises to improve our ability to image geological structures at the scales necessary for accurate assessment of seismic hazard. However, combining the resulting local high-resolution tomography with existing regional models presents an ongoing challenge. We developed a framework based on the level-set method that infers where local data provide meaningful constraints beyond those found in regional models - e.g. the Community Velocity Models (CVMs) of southern California. This technique defines a volume within which updates are made to a reference CVM, with the boundary of the volume being part of the inversion rather than explicitly defined. By penalizing the complexity of the boundary, a minimal update that sufficiently explains the data is achieved.

To test this framework, we use data from the Community Seismic Network, a dense permanent urban deployment. We inverted Love wave dispersion and amplification data, from the Mw 6.4 and 7.12019 Ridgecrest earthquakes. We invert for an update to CVM-S4.26 using the Tikhonov Ensemble Sampling scheme, a highly efficient derivative-free approximate Bayesian method. We find the data are best explained by a deepening of the Los Angeles Basin with its deepest part south of downtown Los Angeles, along with a steeper northeastern basin wall. This result offers new progress towards the parsimonious incorporation of detailed local basin models within regional reference models utilizing an objective framework and highlights the importance of accurate basin models when accounting for the amplification of surface waves in the high-rise building response band.

\section{Plain Language Summary}

Los Angeles is a major city of the United States that is at high risk of damage due to earthquakes, due to the large number of nearby active faults and its location on a deep bowl of weak rock, which tends to amplify earthquake damage. We use a large number of instruments located in Los Angeles district schools to make measurements of earthquakes that occurred near Ridgecrest, California in July 2019. These earthquakes generated a type of energy that is particularly useful for studying the structures responsible for amplification of earthquakes. Using this data, we applied a new imaging technique to create a local model of the northeast Los Angeles basin at higher resolution than had been previously available. Our imaging technique appropriately balances information from previous, lower resolution inversions with the new data obtained in this study.

\section{Introduction}

The Los Angeles (LA) Basin is a deep sedimentary structure whose evolution can be roughly characterized by an initial subsidence and extensional phase during the establishment of the North America - Pacific plate boundary associated with the opening of the Gulf of California and the rotation 
of the Transverse Ranges in the Miocene. This was followed by a period of transpression (Ingersoll \& Rumelhart, 1999), and the generation of a substantial network of thrust faults within the basin (Wright, 1991). In its current state, the basin contains both active strike-slip faults (e.g. the NewportInglewood fault, Whittier-Elsinore fault) and an imbricated stack of blind thrust faults (e.g. the Elysian Park faults, Puente Hills thrust), all of which accommodate the transpressional motion of the basin. These faults contribute to local seismic hazard both by providing source surfaces for earthquakes and by controlling local path effects by shaping the basin geometry (Plesch et al., 2007). The evolutionary history of the LA basin, with ample opportunity to produce and bury organic material during extension followed by the estabilishment of stratigraphic traps during compression, has allowed LA to be a leading producer of oil in the United States (US), helping to fuel a large rise in population during the mid-20 ${ }^{\text {th }}$ century. Development took place predominantly on the soft sediments of the main LA, San Fernando, San Gabriel and San Bernardino basins. As a consequence, LA is both one of the largest and most economically important cities in the US, while also being one of the most exposed to significant earthquake hazard due to the complex fabric of active faults and ground-motion amplifying sedimentary structures associated with the geology that has allowed its preeminence.

Seismic hazard within the basin is controlled by the locations and potential for slip on the multiple local and regional faults of southern California, combined with the significant amplifying effect of the basin on ground motions. The importance of path effects, such as wavefield focusing, multipathing, and basin amplification, on LA basin ground motions has motivated extensive development of seismic velocity models. The ultimate goal of these models is to produce accurate synthetic waveforms at frequency ranges relevant to infrastructure and building codes within the basin. Early efforts focused on creating rule-based models of southern California (Magistrale et al., 1996, 2000) using empirically derived velocity laws (Faust, 1951) in combination with inferred geological structure obtained by correlating surface outcrops, borehole profiles and potential methods (Wright, 1991). Since these inital efforts, regional scale models of southern California have assimilated ever greater quantities of seismic data, including seismic reflection profiles, receiver functions, and earthquake source locations and mechanisms. This increase in the amount of data has led to better demarcated boundaries, including faults (Magistrale et al., 2000; Plesch et al., 2007), and allowed for more lateral variation of within basin velocity structures by using geostatistical methods to tie together disparate seismic data (Süss \& Shaw, 2003; Shaw et al., 2015). Continued development of seismic velocity models of southern California has resulted in two widely used reference Community Velocity Models (CVMs), CVM-S4.26 (Lee et al. (2014), CVM-S hereafter) and CVM-H 15.1.0 (Shaw et al. (2015), CVM-H hereafter), that have incorporated waveform based seismic tomography to further refine the models. CVM-S and CVM-H broadly agree in the positions, average velocity profile, and geometry of the major basins of southern California, however in detail they are quite different, with CVM-H containing more explicit geo- 
a)
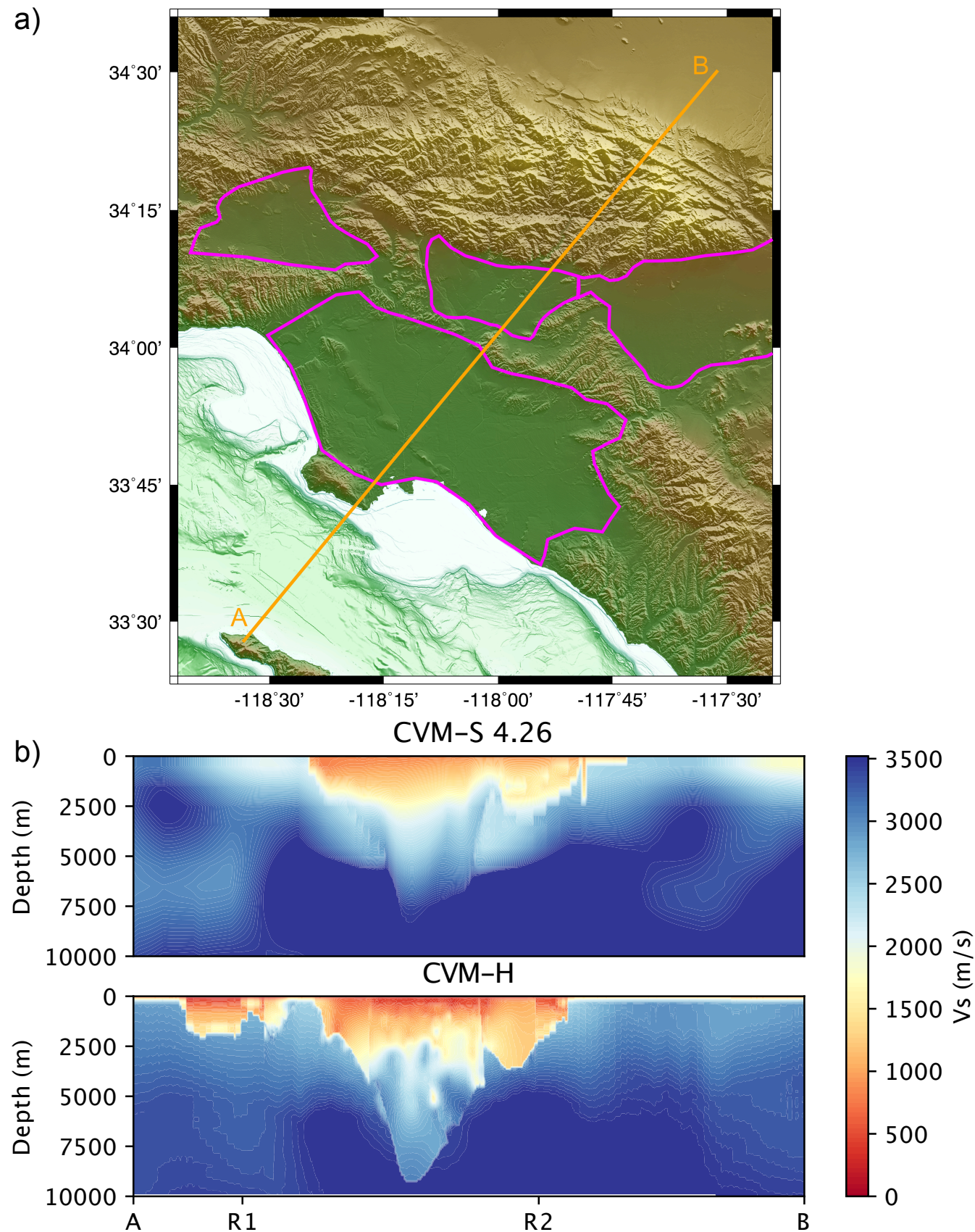

Figure 1. a) Shaded elevation model of southern California showing the outline of the major basins (defined by slope-break analysis) in purple and the transect A-B used for profiles shown in orange. b) Characteristic profiles through the Los Angeles basin for the CVM-S and CVM-H models. Abrupt lateral changes in resolution at positions R1 and R2 are seen in the CVM-H model.

This article is protected by copyright. All rights reserved. 


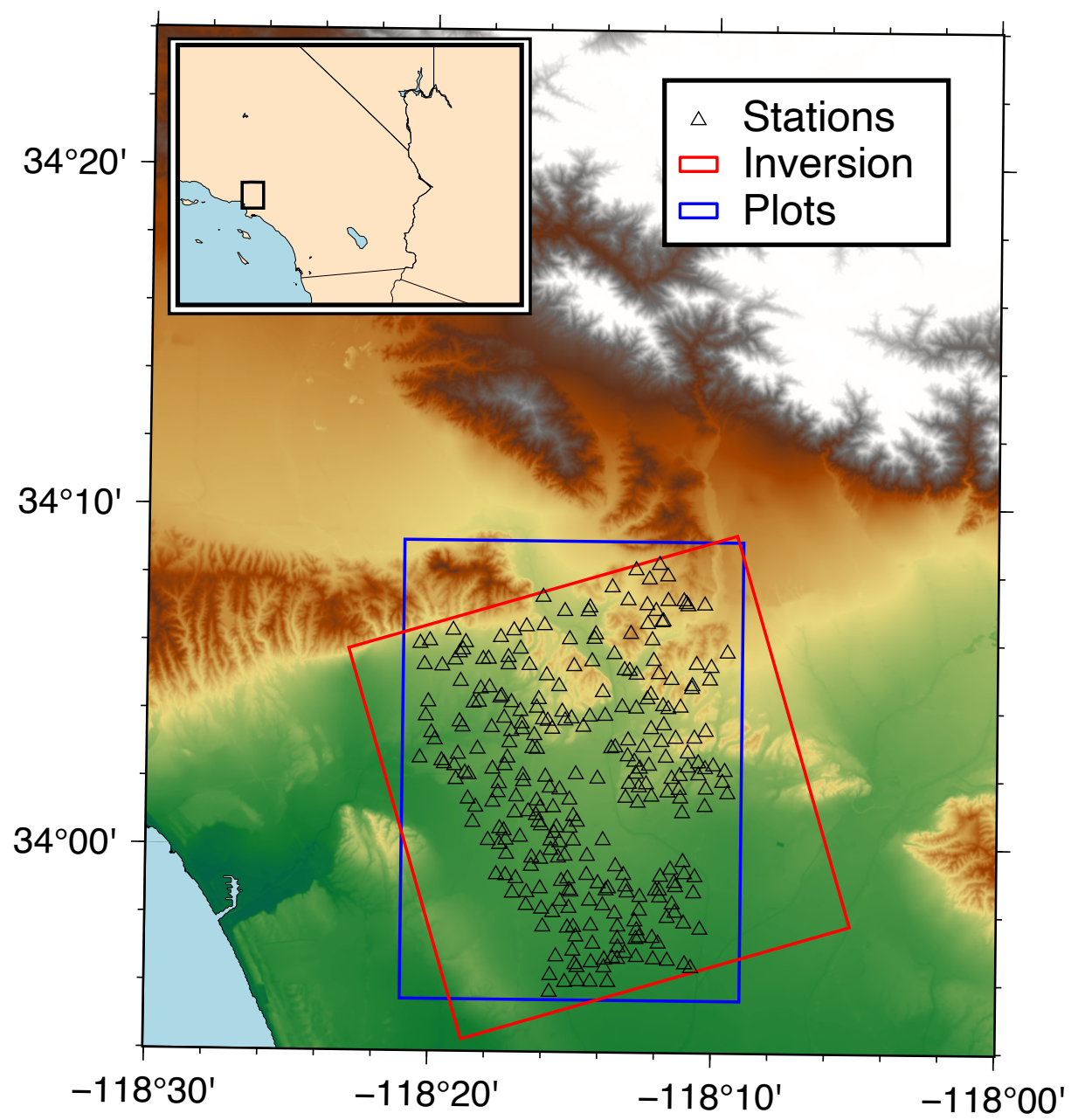

Figure 2. Map of the study region, showing the locations of the CSN stations as open triangles, the boundary of the square inversion region in red, and the boundary of the analysis plots in blue. 
logical information. Figure 1 shows a characteristic cross-section of the LA basin for both models, running from Catalina Island, across the Inner Borderland to Palos Verdes, then through the main LA basin, San Gabriel basin and though the Transverse Ranges to the high desert. This profile makes evident the considerably higher detail present in the CVM-H model due to its construction including explicit geological features (notably including an Inner Borderland basin not present in CVM-S, as seen to the left of profile A-B in Figure 1), as well as its significant artefacts associated with changing lateral resolution, as evident in profile marks R1 and R2. In contrast, CVM-S is significantly smoother than CVM-H due to its reliance on waveformtomography during the final stages of construction, although several sharp resolution based artefacts are also evident, such as the jagged edges of the San Gabriel basin. Many features of the seismic wavefield within the LA basin, such as phase arrival times and P-to-S amplitude ratios, are captured for local events at frequencies of up to $0.2 \mathrm{~Hz}$ (Taborda et al., 2016; Lai et al., 2020). However, excitations of the basin from the recent large regional Ridgecrest earthquake sequence in July 2019 have illustrated that ground motion amplification predictions from finite-difference wave propagation through the SCEC CVM-H and CVM-S models do not accurately predict the observations in the $0.1-1 \mathrm{~Hz}$ range that is relevant for tall buildings within downtown LA (Filippitzis et al., 2021), warranting continued close study of the LA basin velocity model.

Seismic tomography offers the best opportunity for full spatial coverage of the basin at high resolution, especially when dense seismic arrays are utilized. In the southern and central parts of the basin, the deployment of high-density temporary seismic arrays using $10 \mathrm{~Hz}$ corner-frequency geophone nodes by the petroleum industry has enabled considerable exploration of the shallow structure of the basin using ambient-noise derived observables, such as Rayleigh-wave phase velocities, Rayleigh-wave amplifications, and body-wave travel times (e.g. Lin et al. (2013); Bowden et al. (2015); Castellanos et al. (2020); Jia \& Clayton (2021)). However, similarly dense industry deployments have not to date taken place in the northern part of the basin, which encompasses the downtown LA region, with buildings that are highly susceptible to resonant coupling to the basin. The permanent broadband southern California Seismic Network (SCSN), while providing a long time series of excellent quality observations, has already been incorporated into the CVM reference models and does not provide the spatial resolution required for the next generation of basin models. A potential alternative data source is the Community Seismic Network (CSN, Clayton et al. (2012, 2020)), a permanent network of three-component micro-electromechanical system (MEMS) accelerometers, designed to provide real-time strong-groundmotion telemetry in the event of local earthquakes within the LA basin. The CSN instruments have been designed for inexpensive construction, utilizing off-the-shelf components, and have a maximum observable acceleration of $\pm 2 g$, in order to fulfil their primary goal of strong-ground-motion monitoring. As a result, the instrument noise floor is above the amplitude of ground motions produced by smaller regional earthquakes, and is also above the am- 


\section{Data}
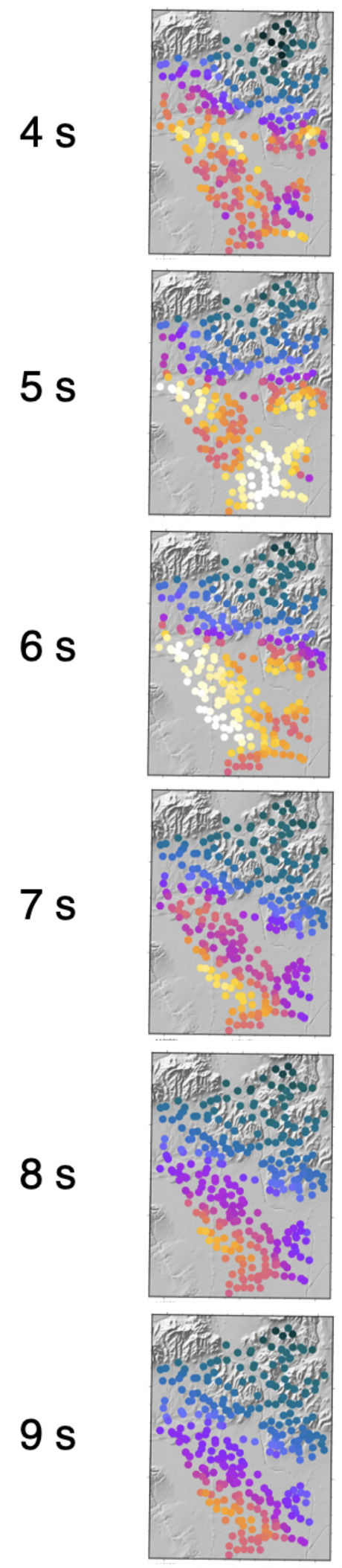

CVM-H
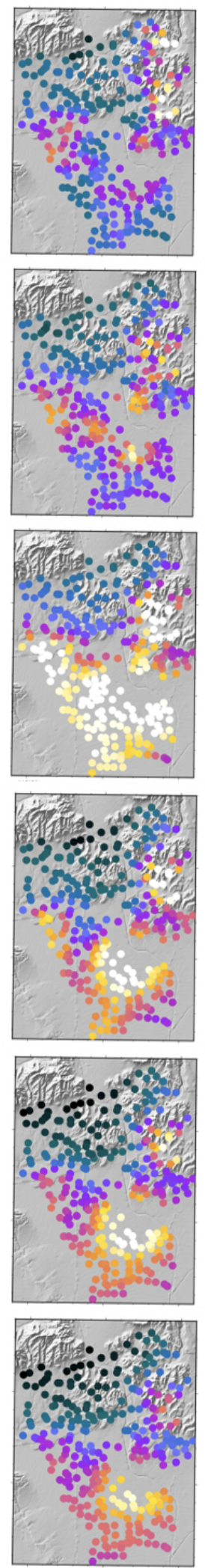

\section{CVM-S}
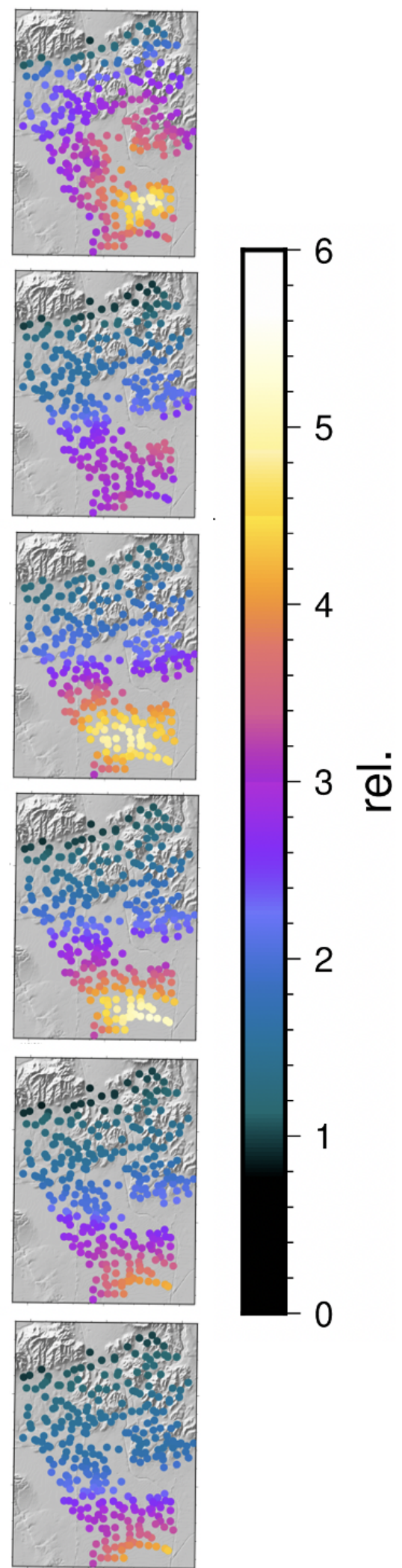

Figure 3. Relative amplification of the maximum amplitude of 3 component pseudo-spectral accelerations (PSA) in the range of 4-9 s from the Mw 7.1 July 52019 Ridgecrest earthquake

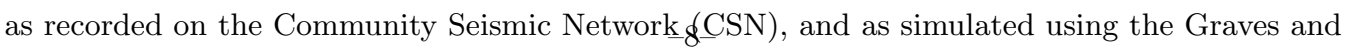
Pitarka rupture generator (Pitarka et al., 2019) and a 3D finite-difference waveform solver for both the CVM-H and CVM-S models (Graves, 1996).

This article is protected by copyright. All rights reserved. 
bient seismic noise level. This unfortunately precludes the use of ambientnoise cross-correlation methods on CSN data as these methods rely on coherent low-level energy propagation between sensors. However, both the $\mathrm{Mw}$ 6.4 and Mw 7.1 2019 Ridgecrest, California earthquakes produced high quality records across the array, allowing for detailed analysis of ground amplification within the basin (Kohler et al., 2020; Filippitzis et al., 2021). The coherent surface-wave energy from these two events, recorded on the CSN, offers a unique opportunity to construct a high-resolution local tomographic model of the northeastern edge of the LA basin. In this study, we use the phase velocity and relative amplitudes of Love waves from both events, along with a 3D surface-wave tomography method based on the level-set method of Muir \& Tsai (2020), to create such a model. The level-set framework extends traditional tomography by allowing for implicitly defined discontinuous interfaces within a velocity model. For instance, Muir \& Tsai (2020) used the level-set method to image the damage zone of the San Andreas Fault at Carrizo Plains using a three-layer model, whereas Tso et al. (2021) presented several applications of the level-set method for developing interpretable block models of electrical resistivity. The ability to handle implicitly defined discontinuities significantly extends traditional tomographic methods, which usually require restrictive and unphysical regularization schemes to be wellposed. We use the level-set method to define a basin volume within which we update a local model - this method allows us to only alter the reference CVM model where we have sufficient data constraints to warrant an update. We take a quasi-Bayesian approach to local updating in which the reference CVM becomes the a priori favored model within the local update. The Love wave data set then updates the CVM prior into an approximate posterior model which includes the influence of both the new data and the data that went into the CVM via its expression in the CVM model. At a global scale, a similar scheme of local quasi-Bayesian model refinement has been proposed by Fichtner et al. (2018), and within the SCEC CVM framework Ajala \& Persaud (2021) have proposed a means of blending local updates into existing regional models - this work differentiates itself by its data-driven choice of model updating region, consistent with estimated data uncertainty. Integration of local models within the SCEC CVM framework will become an important part of hazard modelling within Southern California as high-density arrays allow access to the fine scale detail of path effects. The framework presented in this study represents a parsimonious way to achieve this integration.

\section{Data Collection}

\subsection{Preprocessing}

The data for this study were obtained from the HN accelerometer channels of the Los Angeles Unified School District (LAUSD) subarray of the Community Seismic Network (CSN, Clayton et al. (2012, 2020)), consisting of $200 \mathrm{~s}$ time series after the Mw 6.4 and Mw 7.1 Ridgecrest earthquakes' origin times and recorded at 50 samples/sec. The network is deployed within 


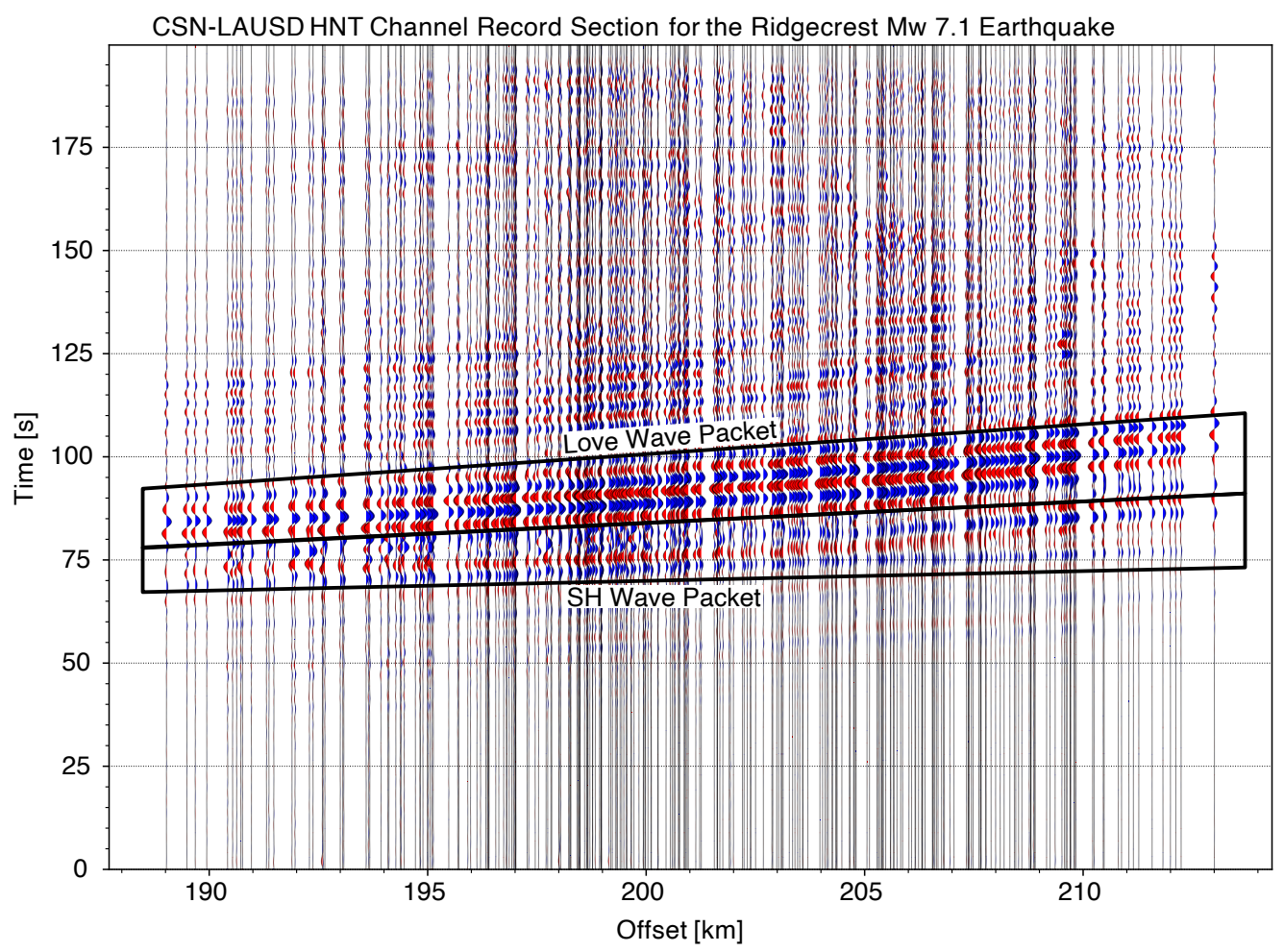

Figure 4. Record Section of the Mw 7.1 Ridgecrest earthquake as recorded on the HNT channel of the CSN-LAUSD array, zero-phase bandpass filtered between 4-10 s. Two main phases are clearly identifiable, with the first arriving phase exhibiting little delay due to the basin at longer offsets, which we infer to be the primary SH arrival. A second, stronger phase, which is delayed by the basin at longer offsets, we infer to be the fundamental Love mode. 
school buildings in the City of Los Angeles, and at the time of the Ridgecrest earthquakes consisted of 300 stations spaced approximately $0.5 \mathrm{~km}$ apart. We used the components of the CSN located within the northeast LA basin, which is the densest part of the array. The study area, including the locations of the stations, is shown in Figure 2. Various display of the Ridgecrest earthquake data are shown in Filippitzis et al. (2021), along with a comparison of the data and predicted ground motions by several methods. For our study, data were first detrended, rotated into the $Z R T$ frame, decimated to $5 \mathrm{~Hz}$ and then detrended once more. Pseudo-spectral accelerations (PSA) were then calculated for both the real data and synthetic 3D finite-difference simulations following the Graves and Pitarka method (Graves \& Pitarka, 2010; Pitarka et al., 2019) for both the CVM-H and CVM-S models by convolving the records with a $5 \%$ damped harmonic oscillator, with the results for 4-9 s period shown in Figure 3. A record section of the high-frequency strong-ground-motion-accelerometer transverse (HNT) channel showing strong $\mathrm{SH}$ polarized phases corresponding to the fundamental Love mode is shown in Figure 4.

\subsection{Love Group Arrival Time and Amplitude Picks}

To make group arrival picks, raw waveforms were first narrow-band filtered at period $P$ using a zero-phase Butterworth bandpass filter with corners at $1 / P \pm 1 /(\sqrt{20} P)$ and then cosine tapered over the first $20 \mathrm{~s}$ of the time series to suppress edge effects. The maximum of the $T$ component envelopes at a central period $P=12.5 \mathrm{~s}$ were set as the first preliminary group arrival pick. The $12.5 \mathrm{~s}$ filtered waveform envelopes were then again cosinetapered with a $6 P$ taper window with $1 P$ edges about this preliminary pick. We then fit a Gaussian function to the waveform envelope, with the center of the Gaussian being used as the finalized group arrival pick at $12.5 \mathrm{~s}$ and the amplitude of the Gaussian being recorded as the Love wave amplitude. Starting with the parameters of the 12.5 s Gaussian as initial values, we then proceeded to work down in $0.25 \mathrm{~s}$ increments on the narrowband filtered waveform envelopes, to a minimum period of $2 \mathrm{~s}$. We tapered with the $6 P$ width cosine around the Gaussian center of the previous period. We then fit a new Gaussian to the shorter-period waveform, initialized using the previous period's Gaussian fit. This method tracks the Love-wave group arrival from long periods, where it is clearly identifiable as the strongest feature, to shorter periods where other features are present. A characteristic example of the group picks is shown in Figure 5.

We took the logarithms of the fitted Gaussian amplitudes and normalized them relative to the mean log at each period to create the amplitude data set. The relatively narrow aperture of the CSN array compared to the distance to the source meant that the geometry was not favorable for traditional tomographic methods. We therefore employed eikonal tomography (Lin et al., 2009, 2014) to calculate surface-wave dispersion curves, which has the additional advantage of naturally handling the curving wavefronts recorded on the CSN, caused by refraction across the basin boundary. Al- 
LAS200
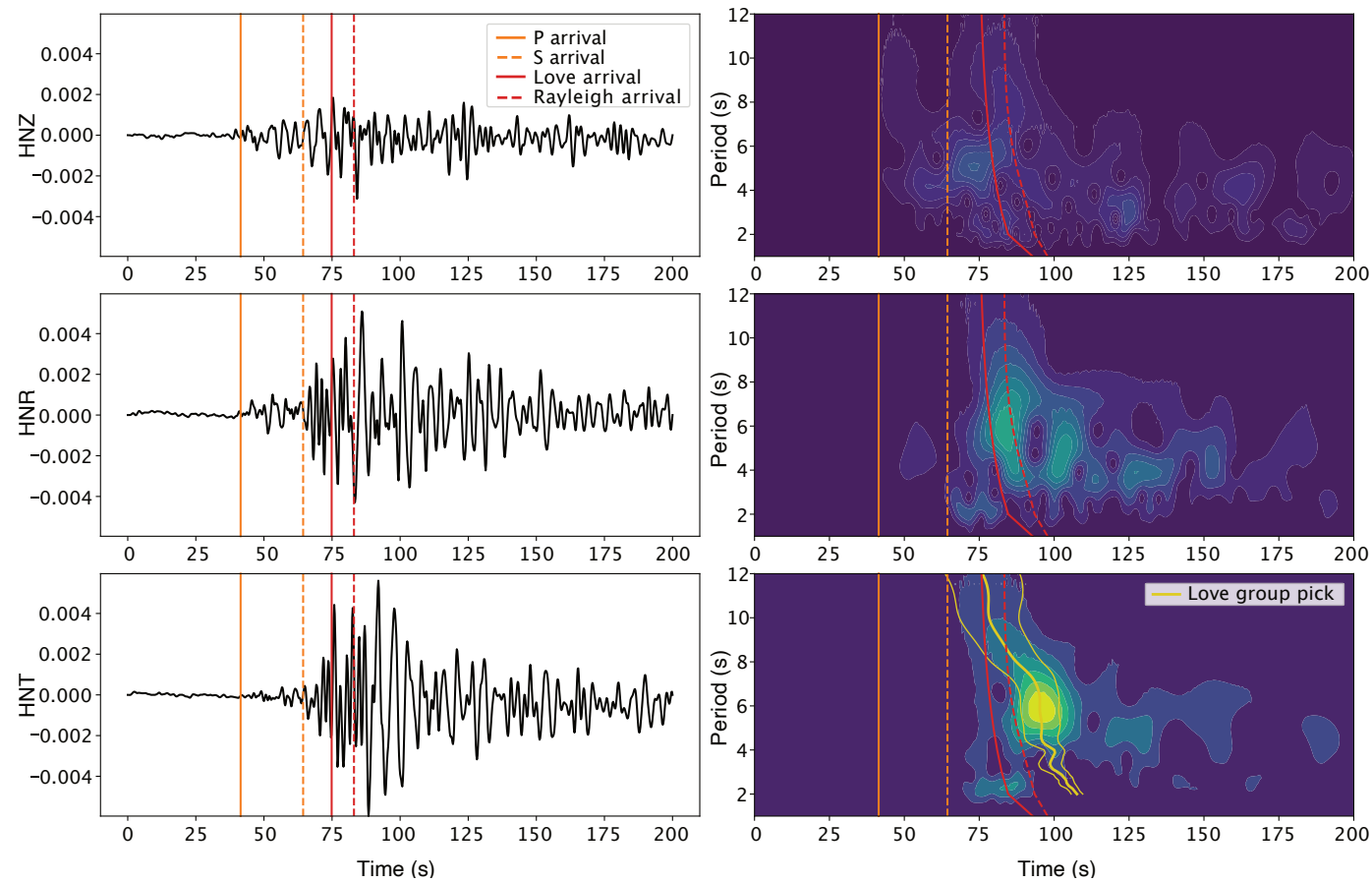

Figure 5. HN waveforms and corresponding continuous-wavelet transform spectrograms for the LAUSD CSN station LAS200 from the July 52019 Ridgecrest Mw 7.1 earthquake. The solid and dashed orange lines show the theoretical arrival times of the $P$ and $S$ waves through the laterally averaged CVM-H model from the hypocentral location to LAS200, and the solid and dashed red lines show the theoretical group arrivals for Love and Rayleigh waves, respectively. All theoretical travel times are offset from the event origin time by $10 \mathrm{~s}$, which is the approximate peak of the USGS moment rate function. The yellow lines show the center and $\pm 1 \sigma$ width of the fitted Gaussian functions to the envelope of the tangential component. The center of these Gaussian functions act as group delay picks for defining the cross-correlation window used for two-station phase delay measurements shown in Figure 6. 
though recent studies (Qiu et al., 2019) have attempted to utilize group arrival times for eikonal tomography of group velocity, there is significant noise associated with the group arrival peak. Furthermore, there are strict conditions on the approximations necessary for using eikonal tomography on group delay times that may not be met when the surface-wave arrival experiences refraction across a basin boundary (Qiu et al., 2019). As such, we did not attempt to utilize group velocity $c_{g}$ in this study, but rather used the group times as a guide for two-station cross-correlation phase delay times as discussed below.

\subsection{Eikonal Tomography from Two-Station Cross-Correlation Phase-Delay Times}

We employ eikonal tomography (Lin et al., 2009) to obtain phase velocity estimates within the densely spaced CSN array. Eikonal tomography obtains phase velocity $c$ directly from the gradient of the phase delay field: $|\nabla \tau| \approx 1 / c$. Eikonal tomography has two principle requirements. Firstly, there must be a clearly identifiable phase delay field $\tau$ (i.e. there is no significant multipathing), a requirement which is met for Love waves in the period range of this study. Secondly, eikonal tomography is derived from an approximation of the transport equation $1 / c^{2}=|\nabla \tau|^{2}-\nabla^{2} A / A \omega^{2}$, where ignoring the amplitude correction is typically taken to be valid for velocity models that are sufficiently laterally smooth that the Laplacian of the amplitude is small. Waves propagating from the Ridgecrest earthquake sequence strike the northeastern edge of the Los Angeles Basin nearly perpendicularly, so any effect of the basin edge on the Laplacian term is limited in extent within the LAUSD-CSN array. It is possible to utilize the full transport equation for determining phase velocity, which is called Helmholtz tomography and may provide improved accuracy if the Laplacian of the amplitude can be accurately calculated (Lin \& Ritzwoller, 2011). For this data set, comparisons between Helmholtz tomography and eikonal tomography show agreement across the basin transition where we would expect the amplitude correction to be strongest, which implies that eikonal tomography is sufficient to capture the correct phase velocity in the center of the array. Spurious values of the Helmholtz tomography solutions occur on the edges of the array due to the difficulty of obtaining accurate values of the Laplacian of the amplitude. Consequently, we limit our data analysis to the phase velocities derived from the eikonal equation as its assumptions appear to be satisfactorily realized and the Helmholtz tomography corrections are not sufficiently robust given our data.

In order to obtain the phase delay field $\tau$ at period $P$ (relative to the northernmost station of the array), we first narrowband filter wavepackets at central period $P$. We then taper the waveform around the group arrival time using a cosine taper with a flat window of width $4 P$ and edges of width $P$. We then calculate the cross-correlation time delay $\Delta \tau_{i j}$ between each pair of stations $i$ and $j$ within a circle of radius $r_{i j}<\max \left(c_{g} P, c_{\min } P\right)$ with a cutoff velocity $c_{\min }=0.5 \mathrm{~km} / \mathrm{s}$. The distance limit reduces the impact of 


\section{Cross-Correlation delay times between nearby stations}

a)

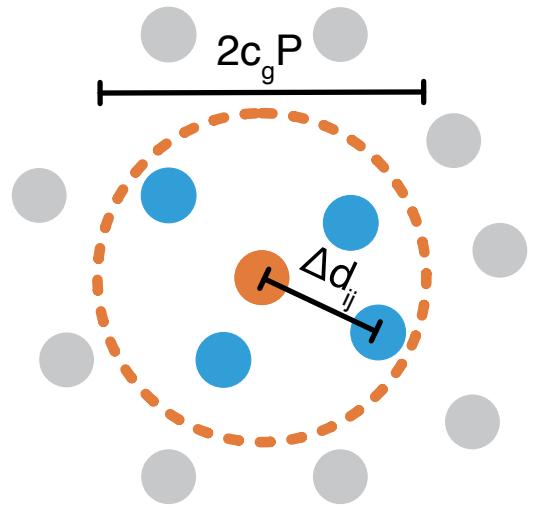

b)

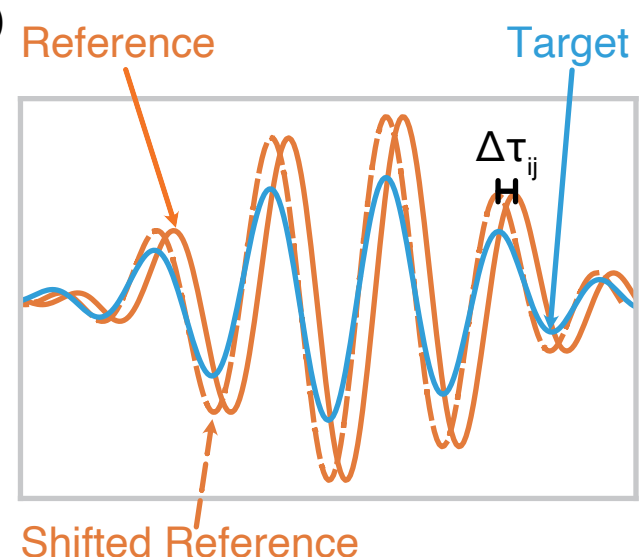

\section{Build graph of delay times and extract Minimum Spanning Tree}

c)

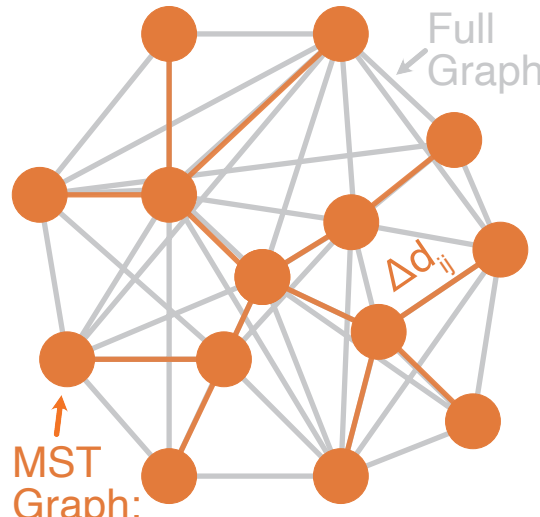

$\min \operatorname{sum}\left(\Delta d_{i j}\right)$ s.t. graph is fully connected

d)

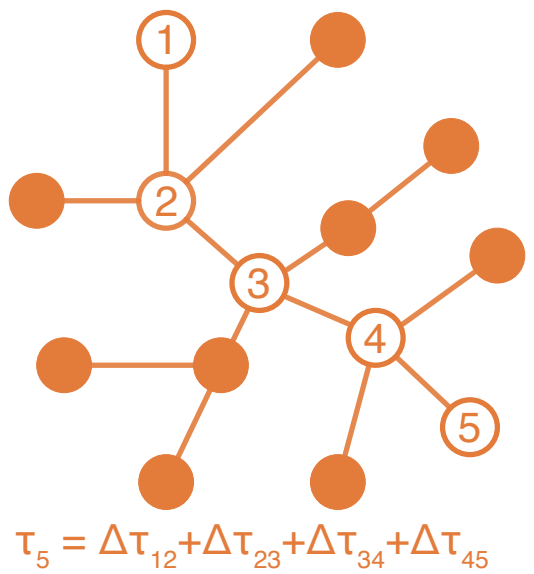

Figure 6. Outline of steps used to construct the phase delay field $\tau$ from narrowband filtered records. In the first two steps, the phase delays between all nearby stations are computed. In a), we draw a circle of radius $r_{i j}<\max \left(c_{g} P, 0.5 P\right)$ and compute the phase delay for maximum cross-correlation, $\Delta \tau_{i j}$, as shown in b). Only nearby stations are used to suppress cycle skipping. In the second phase, we extract the minimum spanning tree (MST) from the graph of collected phase delay times, as shown in c). The MST is a sub-graph that minimizes the total edge lengths (i.e. $\Delta d_{i j}$ ) such that the graph is still fully connected. Finally, in d) we traverse the MST from the northernmost station, summing $\delta \tau_{i j}$ along the edges to get the $\tau$, a minimum-relative-phasedelay surface concordant with the recorded relative phase delays between individual station pairs.

This article is protected by copyright. All rights reserved. 
potential cycle skipping on the phase delay observations, whereas the narrower taper width compared to the group picks also helps to stabilize the cross-correlation calculations. This process is illustrated in Figure 6 a) and b). The relative delays $\Delta \tau_{i j}$ form a graph with stations acting as nodes and the delays acting as edge weights. Similarly, the distances between stations $\Delta d_{i j}$ also form a graph. Appealing to Fermat's principle of least travel time, we extract the minimum spanning tree (MST) of the station distance graph, and then use the geometry of the MST to find an approximate minimum travel time surface. The MST is a unique sub-graph that connects all nodes (stations) with minimum edge weights (distances), with a schematic of this subgraph shown in Figure $6 \mathrm{c}$ ). Summing phase delays $\Delta \tau_{i j}$ along MST edges from the northernmost station gives a minimum relative travel time surface that is concordant with the observed phase delay data, as shown in Figure $6 \mathrm{~d}$ ). We also tested MSTs extracted from both the graph of normalized crosscorrelation values, as well as the graph of phase delays themselves, but found that the MST based on distance weighting gave the best performance in the final phase velocity maps. We then smooth the travel-time surface at each period by first fitting a high-tension cubic spline to the data, removing all outlying data points for which the fit residual at that point was greater than one standard deviation of all collected residuals, and then refitting the spline to the remaining data. This outlier removal cleans the phase delay dataset of any remaining cycle-skipped measurements. This smoothed surface $\tau$ is then used to calculate phase velocity $c$ at period $p$ using the eikonal equation $|\nabla \tau|=1 / c$.

\subsection{Estimating Measurement Uncertainty}

The only available earthquakes that have produced sufficiently strong ground motions to record at least one octave of frequencies of Love waves are the Mw 6.4 and Mw 7.1 Ridgecrest events. Two events are insufficient to obtain useful statistical estimates of measurement uncertainty using only data recorded at individual stations. However, given that the surface-wave measurements have a finite area of sensitivity that overlaps substantially between neighbouring stations, we can approximate the measurement uncertainty at a point by including all data within the sensitivity area. To calculate this, we bin data statistics over subarrays of radius $\lambda / 4$ to obtain an estimate of the measurement uncertainty, where $\lambda$ is the fundamental Love wavelength at the period of measurement and the station of interest. At station $i$, we calculate the mean of the relative log amplitude $\tilde{a}^{i}=\left(a_{6.4}^{i}+a_{7.1}^{i}\right) / 2$ and phase velocity $\tilde{c}^{i}=\left(c_{6.4}^{i}+c_{7.1}^{i}\right) / 2$ where $a_{6.4}$ and $c_{6.4}$ are the amplitude and phase velocities for the $\mathrm{Mw} 6.4$ earthquake, respectively, and likewise $a_{7.1}$ and $c_{7.1}$ are the amplitude and phase velocity for the Mw 7.1 earthquake. We then estimate the $1 \sigma$ uncertainty in the mean by averaging over the data variance at nearby stations:

$$
\begin{aligned}
\sigma_{a}^{i} & =\sqrt{\sum_{j \in d_{i j} \leq \lambda / 4}\left(a_{6.4}^{j}-\tilde{a}^{j}\right)^{2}+\left(a_{7.1}^{j}-\tilde{a}^{j}\right)^{2}} / \sqrt{2} \\
\sigma_{c}^{i} & =\sqrt{\sum_{j \in d_{i j} \leq \lambda / 4}\left(c_{6.4}^{j}-\tilde{c}^{j}\right)^{2}+\left(c_{7.1}^{j}-\tilde{c}^{j}\right)^{2}} / \sqrt{2}
\end{aligned}
$$


where $d_{i j}$ is the distance between stations $i$ and $j$. The uncertainty correlation matrix $P_{i j}$ is modeled using a squared-exponential covariance function with characteristic length scale equal to one quarter of the average Love wavelength at predicted at stations $i$ and $j$, which accounts for spatially correlated uncertainty, with the addition of a diagonal term to account for uncorrelated uncertainties

$$
P_{i j}=\delta_{i j}+\exp \left(-8 d_{i j}^{2} /\left(\lambda_{i}+\lambda_{j}\right)^{2}\right),
$$

where $\delta_{i j}$ is the Kronecker delta. For each period the empirical uncertainty covariance matrices are therefore given by $\Gamma_{c}=\sigma_{c} P \sigma_{c}^{T}$ and $\Gamma_{a}=\sigma_{a} P \sigma_{a}^{T}$ where $\sigma_{c}$ is the collected vector of individual station phase-velocity uncertainty measurements across all periods, and $\sigma_{a}$ is likewise the vector of amplitude uncertainty measurements. Future work on uncertainty modelling could account for a variable scaling between the diagonal and non-diagonal terms in $P$, and model the correlations between measurements at neighboring periods; however for reasons of computational expediency we do not develop these analyses here.

\section{Inversion Methodology}

\subsection{Model Parameterization}

Having obtained measurements $\tilde{c}$ and $\tilde{a}$ and associated data uncertainty matrices $\Gamma_{c}$ and $\Gamma_{a}$ for phase velocity and log-relative amplification within the CSN, we are now in a position to model them and invert for a local basin update. We seek to obtain a parsimonious local update that balances the constraints of new, densely recorded data, with the already well developed models presented in the SCEC CVMs. Ideally, we would perform a fully Bayesian inversion taking a CVM as a prior model; however as robust model uncertainties for the CVMs are not available, this approach would be highly dependent on subjective estimates for setting the prior, and would furthermore be extremely computationally expensive for the nonlinear forward models required to predict our recorded data. Instead, we recognize that the sensitivity of our data is highly contained within the basin itself, given the characteristic phase velocities $c$ and periods $p$ of our study and the heuristic sensitivity depth of $c p / 4$ for Love waves in a power-law basin-style velocity profile, given by Haney \& Tsai (2020). Taking advantage of the Love wave sensitivity being largely restricted to the basin, we utilize the level-set-tomography framework of Muir \& Tsai (2020) to explicitly define a volume within which we perform our model updates as part of the model parameterization. The level-set method implicitly defines boundaries within a domain by taking them to be a contour interval of a function on that domain (Osher \& Sethian, 1988; Gibou et al., 2018). For example, the basin boundary (a 2D surface) may be defined by the zero-contour of a continuous 3D function. The roughness and topology of the interface can be controlled by the properties of the underlying function. In our case, by appropriately regularizing the boundary of the inversion volume, we achieve the desired parsimony between the a priori CVM model and constraints from our newly observed data. 


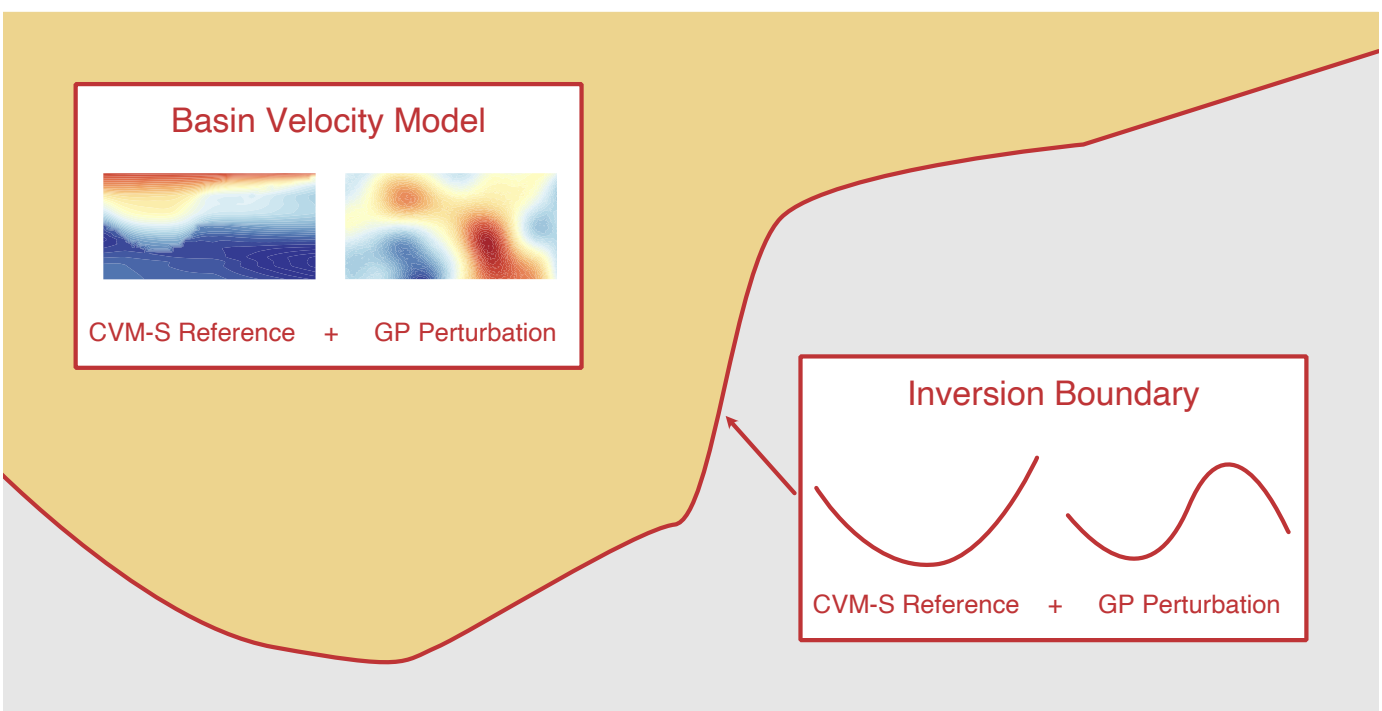

Figure 7. Schematic of the model definition, showing the construction of the velocity model update and the boundary of the inversion, both constructed from a CVM-S reference perturbed by a Gaussian Process. The background model, schematically shown in grey, is given by the unaltered CVM-S model.

In this study, our model parameterization consists of two parts - a boundary to the inversion domain, and the velocity perturbations within that domain. Both components of the model are given by Gaussian Processes (GP) (Rasmussen \& Williams, 2006). GPs are a general method of introducing spatial relationships into spatial interpolation, projection and inverse problems (Valentine \& Sambridge, 2020a,b). In this study we use GPs to regularize our inversion, in an analogous way to the spatial damping and smoothing used in the frequently used Tikhonov regularization framework (Aster et al., 2018), although the the smoothing induced by GPs is more flexible and easier to interpret. GP models are defined by the property that, for a collection of sample points $x$, the output $f(x)$ of the GP is jointly distributed as a multivariate normal distribution. The wide range of choice in defining the covariance matrix of the multivariate normal makes the GP modelling framework very powerful. For instance, nearly diagonal matrices result in highly uncorrelated spatial behaviour, where only the amplitudes of the output $f(x)$ are affected. Matrices with large off-diagonal components can introduce interesting spatial covariances in $f(x)$, such as restricting the output to be smooth up to certain derivatives, include spatial periodicity, prefer correlation at certain length scales etc.

The pairwise covariance between $f(x)$ and $f\left(x^{\prime}\right)$ is given by a covariance function $C\left(x, x^{\prime}\right)$. Given that the covariance function controls the roughness, characteristic length scale(s) and potential periodicities of the GP, the selection of an appropriate covariance function is the most important part of GP modelling. We use a Whittle-Matérn covariance function in this study, which is a common choice for initial treatment of spatial modelling. The 
Whittle-Matérn covariance allows explicit control over the degree of roughness, ranging from not-differentiable to infinitely smooth depending on a parameter $\beta$. The spatial correlations of Whittle-Matérn GPs have a single dominant length scale $l$. The Whittle-Matérn covariance function is given by

$$
C\left(x, x^{\prime}\right)=\sigma^{2} \frac{2^{1-\beta}}{\Gamma(\beta)}\left(\frac{\left\|x-x^{\prime}\right\|_{2}}{l}\right)^{\beta} K_{\beta}\left(\frac{\left\|x-x^{\prime}\right\|_{2}}{l}\right),
$$

where $\Gamma$ is here the gamma (or extended factorial) function and $K_{\beta}$ is the modified Bessel function of the second kind. A comprehensive treatment of classical GP models, including discussion of other common choices of covariance functions, may be found in Rasmussen \& Williams (2006). The statistical properties of a GP are controlled by its hyperparameters, which for the Whittle-Matérn covariance function are $l$, the characteristic length scale, $\sigma$ the characteristic scale of perturbations, and $\beta$ the regularity parameter. Individual realizations of GPs using the Whittle-Matérn covariance are $\beta-$ $\frac{1}{2}$ times continuously differentiable. In practice $\beta$ is very hard to infer in most inverse problems as finite observations are unable to resolve rough details, and so it is set to $\beta=3 \frac{1}{2}$ for the remainder of this study. This choice of $\beta$ generates sufficiently smooth models to ensure that Love-wave eigenvalues are correctly calculated, and does not introduce any artificial roughness into samples from the posterior distribution that is not warranted by the data. We do not set $\beta$ to any higher value (which would result in greater smoothness) so that the basin boundary can be sufficiently steep to capture the abrupt change in Love wave amplification.

GP models with variable hyperparameters offer great flexibility, however they are expensive to compute in the spatial domain as they require repeated inversion of the spatial prior covariance matrix $C$, which is a function of the hyperparameters. The inversion of this dense matrix is in general an operation of complexity $O\left(n^{3}\right)$ for $n$ model evaluation points. To accelerate the GP computations, rather than evaluating the GP at each station and forward model depth grid-point, we approximate the model by defining it on a regular grid with $n_{\text {cell }}$ grid nodes in each dimension. Using a structured grid allows us to specify the model by means of its hyperparameters and 3D Fourier coefficients $\xi_{v}$ and $\xi_{b}$ for the velocity and inversion boundary components respectively, as is further discussed in the Appendix (Lindgren et al., 2011; Chen et al., 2019). Efficient sampling of the GP can then be performed by an inverse real Fast Fourier Transform (complexity of order $O\left(3 m^{3} \log (m)\right)$ where $\left.m=n_{\text {cell }} / 2+1 \ll n\right)$, followed by interpolation by cubic splines to the station locations required for computing the forward model for phase velocity and amplitude underneath each station. We use the same length scale parameter $l$ for both the velocity update and the inversion boundary; the inversion domain is $22 \times 22 \times 12 \mathrm{~km}$ in size, which must be rescaled to a unit cube for the inverse Fourier transform. The inversion area was determined by finding the smallest square that encompassed the stations, and is shown in Figure 2. We use 16 cells in each dimension, and a rescaled $\tilde{l}$ parameter on the unit cube domain, which induces an effective length scale of $l_{x y} \sim 22 \tilde{l}$ in the horizontal direction and $l_{z} \sim 12 \tilde{l}$ 
in the vertical direction - equivalent to assuming vertical heterogeneity has a characteristic length scale half that of lateral heterogeneity. We denote the evaluation (via inverse FFT) of the velocity GP model given velocity Fourier coefficients $\xi_{v}$, length scale $\tilde{l}$ and velocity characteristic perturbation amplitude $\sigma_{v}$ at a location $(x, y, z)$ by $G P V_{\xi_{v}, \tilde{l}, \sigma_{v}}(x, y, z)$, and the evaluation of the inversion boundary given boundary Fourier coefficients $\xi_{b}$, lengthscale $\tilde{l}$ and boundary characteristic perturbation amplitude $\sigma_{b}$ at a location $(x, y)$ by $\operatorname{GPB}_{\xi_{b}, \tilde{l}, \sigma_{b}}(x, y)$. For both GP models, a Whittle-Matérn covariance function is assumed. We use the CVM-S velocity and basin profile as the reference model which we will perturb during the inversion, to ensure initialization near a physical solution. CVM-S was chosen over CVM-H as the reference due to its smoothness, which lends itself to more concordant velocity models across the inversion boundary, and also because it better fits waveforms within the basin (Lai et al., 2019).

The $V_{s}$ model is therefore given by

$V_{s}(x, y, z)= \begin{cases}V_{\mathrm{CVM}-\mathrm{S}}(x, y, z)+G P V_{\xi_{v}, \tilde{l}, \sigma_{v}}(x, y, z) & z<z_{\mathrm{CVM}-\mathrm{S}}(x, y)+G P B_{\xi_{b}, \tilde{l}, \sigma_{b}}(x, y) \\ V_{\mathrm{CVM}-\mathrm{S}}(x, y, z) & z \geq z_{\mathrm{CVM}-\mathrm{S}}(x, y)+G P B_{\xi_{b}, \tilde{l}, \sigma_{b}}(x, y),\end{cases}$

where $V_{\mathrm{CVM}-\mathrm{S}}$ and $z_{\mathrm{CVM}-\mathrm{S}}$ are the reference $\mathrm{S}$ velocity model and basin edge extracted from CVM-S. CVM-S does not explicitly define a basin edge, and so we discuss how we define the reference basin geometry in Section 3.2. A graphical schematic of the definition of the discretized model is shown in Figure 7. Density and $V_{p}$ are then calculated from the $V_{s}$ model using the empirical relationships of Brocher (2005), which are suitable for basins within southern California.

\subsection{Extracting Reference Basin Depth Profiles from CVM- $\mathrm{S}$}

The SCEC CVM-S model is defined by a gridded voxel parametrization of $V_{P}, V_{S}$ and $\rho$, i.e., it does not contain explicit definitions of basin boundaries. To obtain reference boundaries for the CVM-S model, we utilized the following procedure. At each depth slice, we computed the mean and standard deviation of $V_{S}$. We then flagged each voxel for which $V_{S}$ was slower than one standard deviation below the mean velocity of that depth slice as a potential basin candidate. For each 1D depth profile, we then worked from the second $(\mathrm{z}=500 \mathrm{~m})$ depth slice downwards, flagging a voxel to be within a basin only if all voxels above it were also flagged. Working from the second depth slice avoids the connection of individual basins due to the large low velocity surface feature in the CVM-S 4.26 model.

This process assumes that basins are strictly convex, which is not true in general but is a useful approximation to begin the inversion process. Using the scipy module ndimage (SciPy 1.0 Contributors et al., 2020), we then performed image segmentation using the label function. This function assigns each connected volume a unique integer index, that can then be used to extract the basin from the larger regional velocity model. This process 


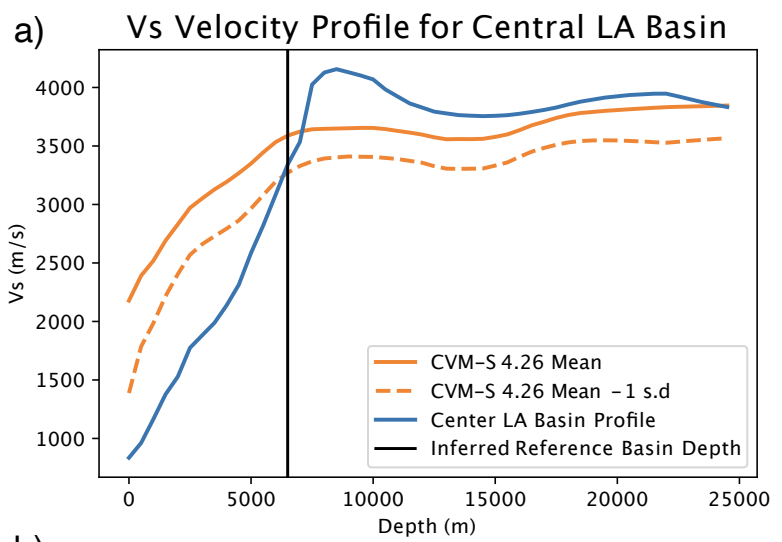

b) Inferred Candidate Basin Depths

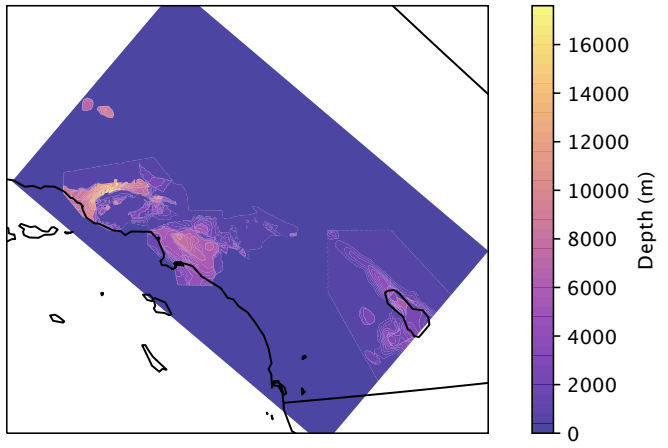

c)

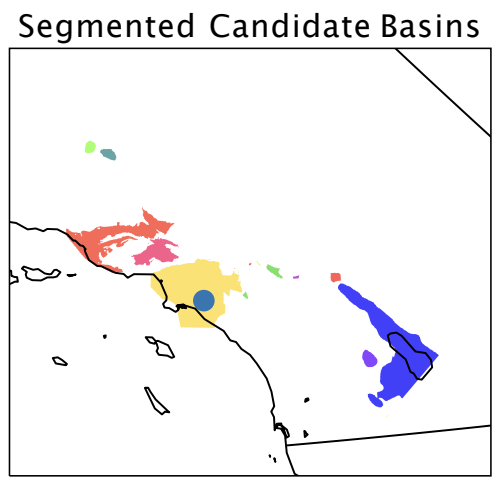

Figure 8. Outline of steps used to extract a reference basin surface from CVM-S. a) For each vertical profile in CVM-S, we determine where (if anywhere) the $V_{S}$ profile first becomes faster than one standard deviation below the mean CVM-S velocity at that depth. All depths above this level are set to be a potential candidate basin at the location of the profile. In b), we show the extracted candidate basin depths across southern California. In c), we strip off the top 500 $\mathrm{m}$ (which is highly connected) and then use the SciPy ndimage label function to segment the remaining data volume by assigning each independent connected volume a unique index. The three major basin families of southern California are clearly seen in pink (Ventura / San Fernando), yellow (Los Angeles / San Gabriel / San Bernardino) and blue (Salton Trough). 
Love-wave amplification

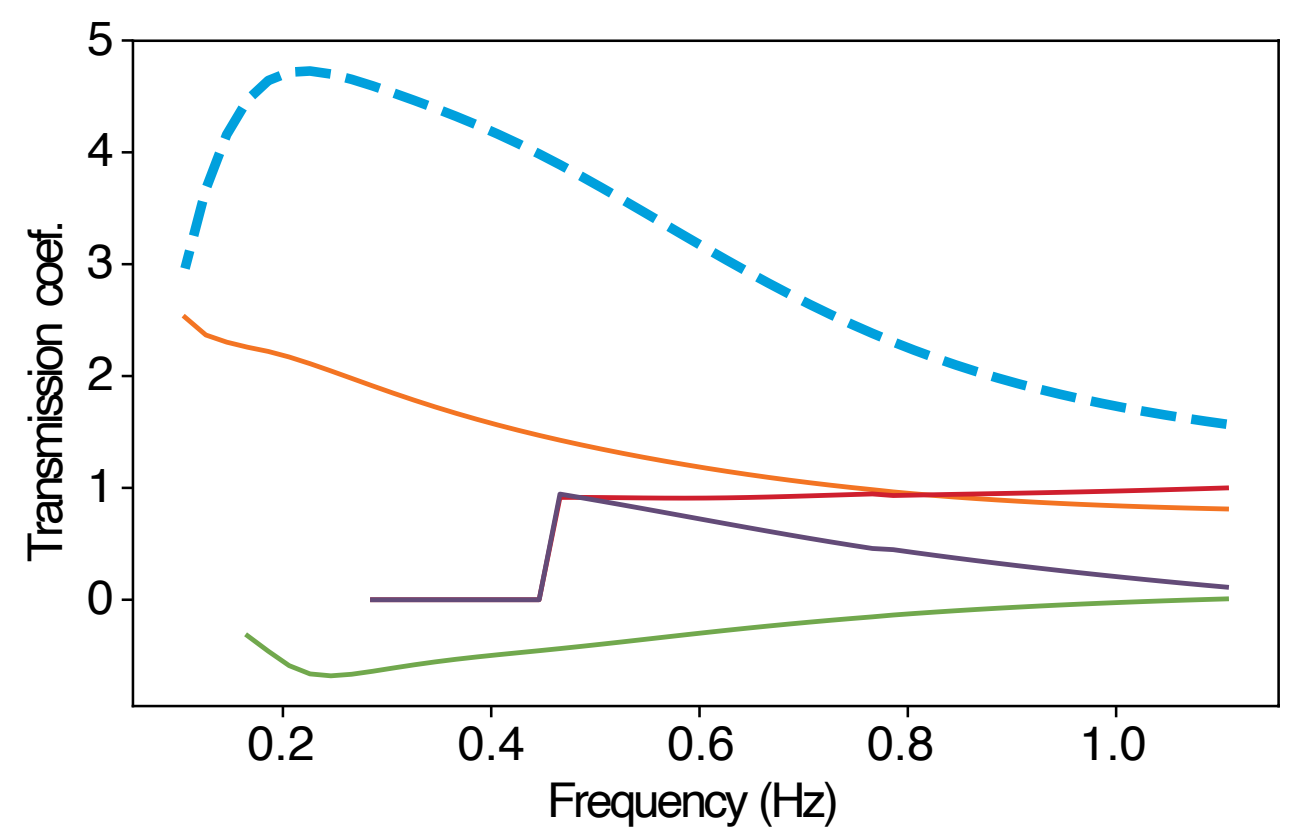

Figure 9. Transmission coefficients for a Love wave entering the Los Angeles basin obtained using a 1D mode-coupling theory (Datta, 2018; Brissaud et al., 2020). This represents a worstcase mode-conversion scenario, with the true basin exhibiting a smoother horizontal gradient and hence less conversion. Even in this case, the conversion of energy from the fundamental mode to first overtone $T_{01} / T_{00}$ is relatively small, suggesting that our use of classical Love-amplification theory is appropriate.

In order to predict the data from the final rasterized velocity model given by our model parametrization, we employ the lumped-mass finite element method for surface-wave eigenvalue calculation first proposed by Lysmer (1970), and implemented for Love waves by Haney \& Tsai (2020). The rasterized model is interpolated onto a set of finite elements of exponentially increasing thicknesses $h$ given by $h_{n}=a \min (\lambda) * \exp (N /(n a)) / n$ where $N=50$ is the number of layers in the model, $\min (\lambda)$ is the minimum wavelength 
corresponding to the minimum phase velocity in a reference model, and $a=$ 0.25 is the constant used to control the exponential scaling. This exponential scaling heuristically balances the need for finer resolution near the top of the model when calculating shorter period Love waves, against computational efficiency, in a way that is near optimal due to the approximate exponential shape of Love eigenfunctions (Tsai \& Atiganyanun, 2014; Haney \& Tsai, 2015, 2017, 2020). These layers are stacked on top of 4 layers of thickness $h=10 \mathrm{~km}$ simulating an infinite half-space to avoid contamination with the locked lower boundary condition. We then set up the finite element stiffness and mass matrices as given by Haney \& Tsai (2020), and solve for the maximum slowness eigenfunction $u$ that corresponds to the fundamental Love mode as well as the phase velocity $c$ and group velocity $c_{g}$. The relative amplification of Love waves directly observed between two locations can then be calculated by

$$
\frac{a_{1}}{a_{2}}=\left(\frac{c_{g_{1}} I_{1}}{c_{g_{2}} I_{2}}\right)^{-1 / 2},
$$

with $I=\int_{0}^{\infty} \rho(z) u(z)^{2} d z$ (Bowden \& Tsai, 2017; Bowden et al., 2017). Transmission coefficients obtained using a 1D mode-conversion theory (Datta, 2018; Brissaud et al., 2020), applied to Love waves transmitting from a characteristic out-of-basin velocity and density profile to an in-basin profile, are plotted in Figure 9. The results of this mode-conversion test suggest that any potential modelling error from neglecting mode-coupling is small. As we use a derivative-free inversion method, these quantities are sufficient to solve for the optimal model.

\subsection{Inverse Solver}

We use an extension of the Ensemble Kalman Sampler (EKS, GarbunoInigo et al. (2020)) to perform the inversion. This method uses an interacting ensemble of particles that follow Langevin diffusion dynamics to infer a Gaussian approximation to the posterior of the inverse problem. The EKS is derivative-free and embarrassingly parallel in the forward model, which enables rapid user iteration between different datasets and forward modelling methods, as well as easy deployment on heterogenous computing networks. The EKS as outlined in Garbuno-Inigo et al. (2020) assumes that all model parameters have a Gaussian prior. This restricts the model to have fixed hyperparameters (e.g. $\tilde{l}, \sigma_{v}, \sigma_{b}$, as required to set the statistical behaviour of the model parameterization described in Section 3.1), which introduces a significant potential for practitioner bias as we do not have a good basis for estimating these a priori. Consequently, we have further developed the EKS to handle hierarchical models with variable hyperparameters. The original EKS and our extension to it are discussed in detail in Appendix A. The priors for the velocity hyperparameters are given by $1 / \tilde{l} \sim \operatorname{Normal}(0,0.6)$ and $\sigma_{v} \sim \operatorname{Normal}(0,0.1)$ in scaled inverse $\mathrm{km}$ and $\mathrm{km} / \mathrm{s}$ respectively. Experimentation has shown that the characteristic boundary perturbation amplitude $\sigma_{b}$ is not sufficiently identifiable from our data, so we set it to a reasonable value of $0.5 \mathrm{~km}$ that is small enough to avoid large, unrealistic changes 


\section{EKS Convergence Diagnostics}

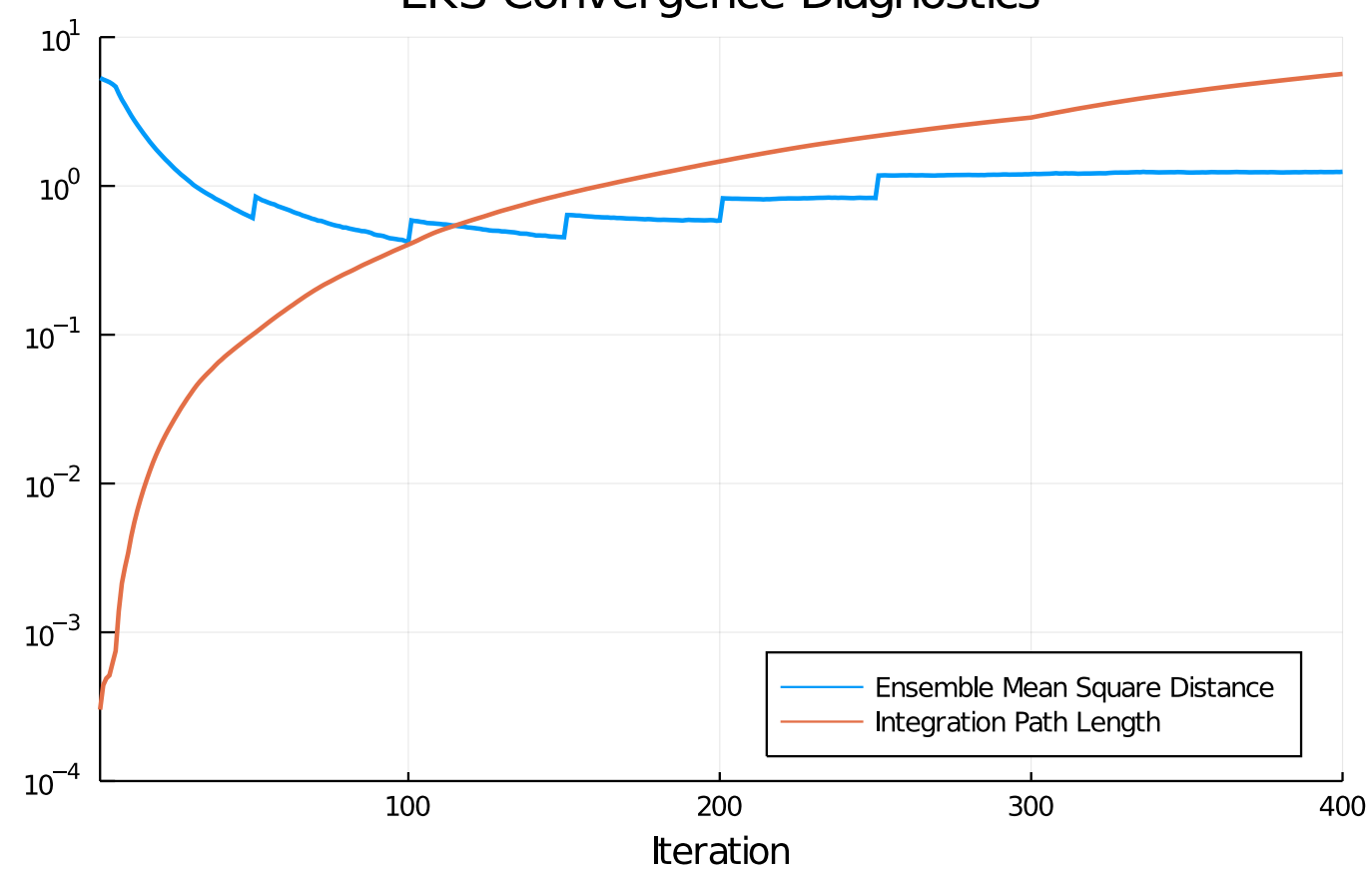

Figure 10. Convergence diagnostics of the Ensemble Kalman Sampler (EKS) showing the Mean Square Distance between ensemble members converging to a constant, which suggests the ensemble has reached an equilibrium and is approximating the posterior. The integration path length steadily increases, showing that the ensemble is not being forced to take very small steps (heuristics from Garbuno-Inigo et al. (2020) suggest a path length of 2 is sufficient to approximate the posterior). 
a)

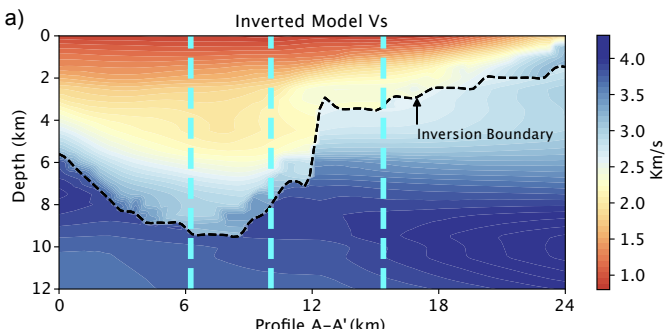

b)

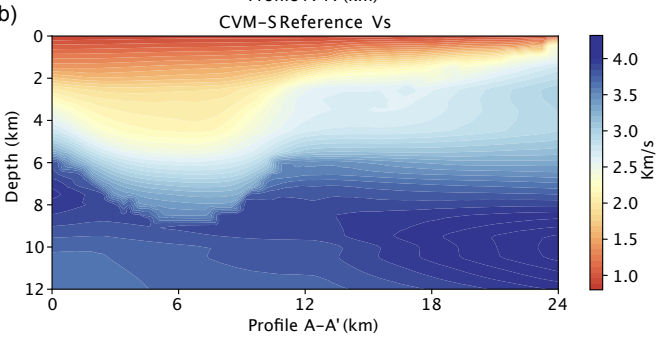

c)

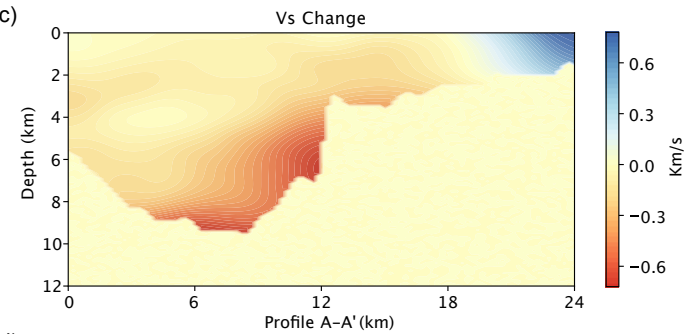

d)

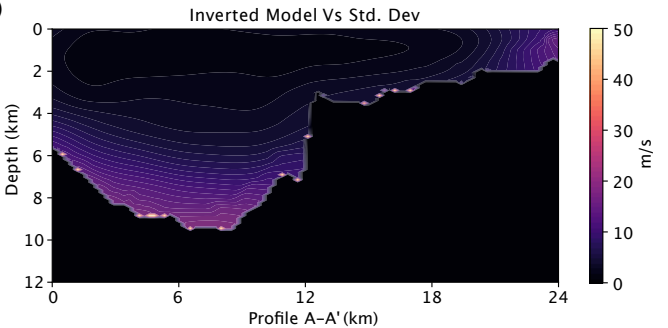

Figure 12. a) Mean of the final ensemble $V_{S}$ model, b) CVM-S reference model $V_{S}$, c) difference between final model and reference model, d) standard deviation of the final ensemble $V_{S}$ model.

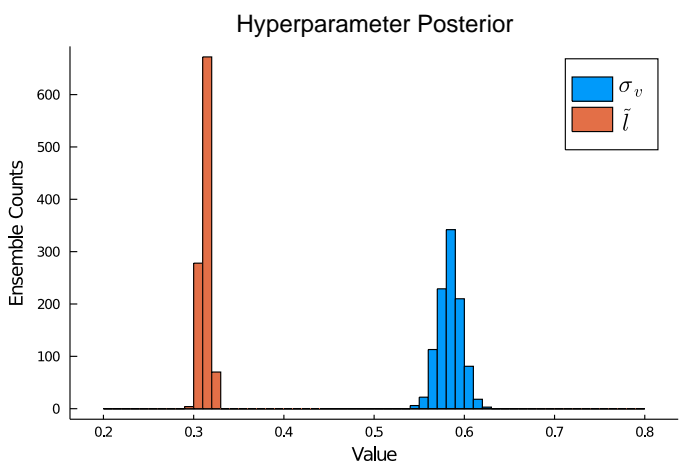

Figure 13. Approximate posterior distribution from the final ensemble for the hyperparameters $\tilde{l}$ and $\sigma_{v}$. 


\section{Output Model Profiles}
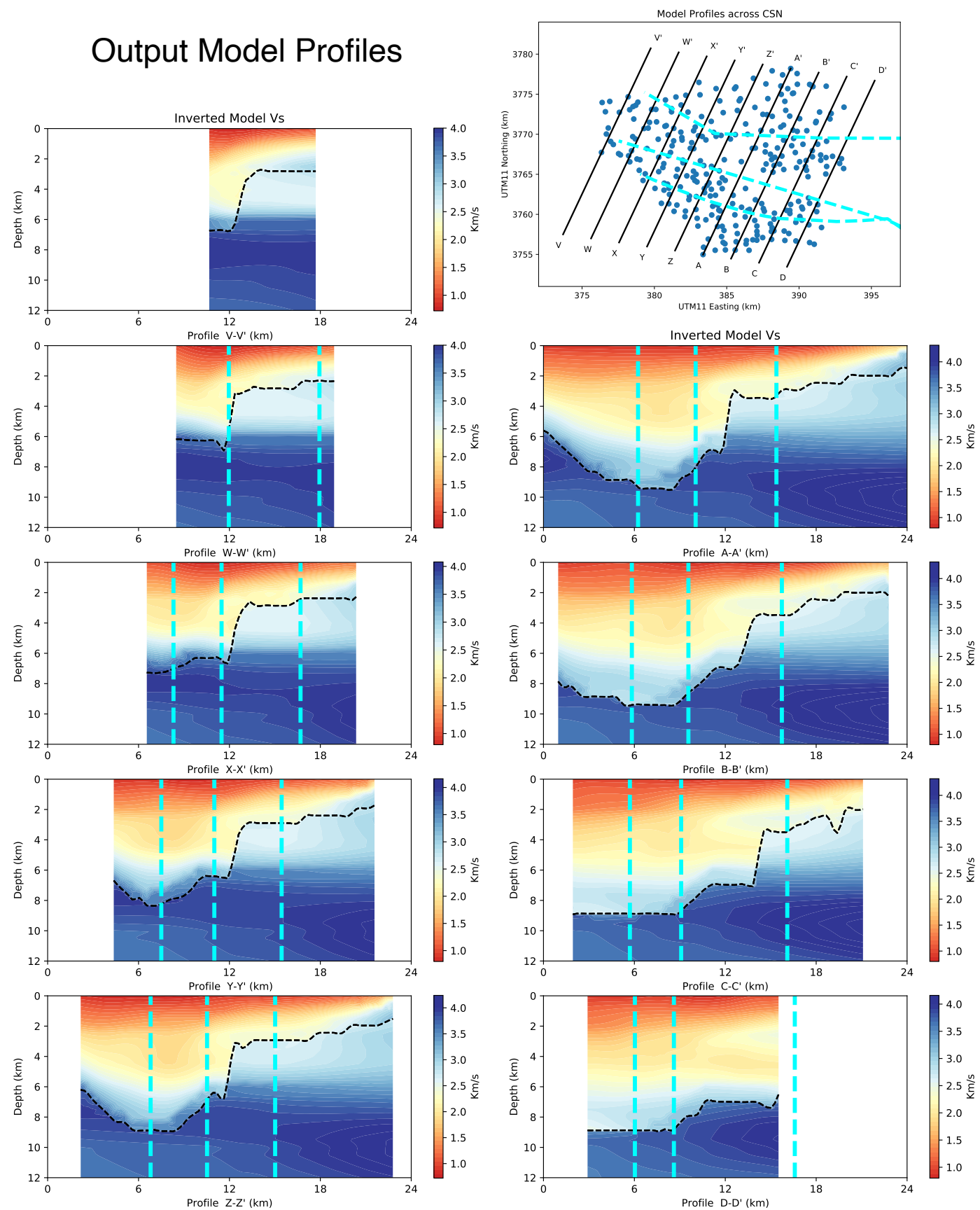

Figure 14. Profiles of the mean output $V_{s}$ across the Los Angeles Basin, with inferred Quaternary faults in dashed cyan and the inferred edge of the inversion shown in dashed black. 
extracted from CVM-S in Section 3.2, and the depth to the same velocity contour in the final model. Figure 12 shows the details of the inversion along profile A-A'. Figure 13 shows the approximate posterior distribution of the hyperparameters in the inversion. In Figure 12, we also show the reference CVM-S model used to initalize the inversion, the mean of the EKS ensemble, the difference between these two, and the standard deviation of the ensemble. The standard deviation gives a sense of the relative uncertainty of the final inversion. As discussed in Garbuno-Inigo et al. (2020), in the lowparticle limit EKS sampling cannot fully capture the range of uncertainty in the true inversion posterior, and so the plotted standard deviations are best assessed in a qualitative fashion. The EKS ensemble indicates that the highest uncertainties are along the boundary of the model. Within the inverted area of the final model, the uncertainties are highest in the deep central basin where the 4-10 s Love wave period range offers less sensitivity, and near the northeastern edge of the model where the phase velocities are high, resulting in small travel time gradients and hence higher uncertainties when employing eikonal tomography.

There are two principle features that are apparent from the results of the inversion. The first and most significant finding is that the data support a deeper Los Angeles basin along its northeastern edge, with an especially large jump in basin depth in the area immediately abutting the Upper Elysian Park fault as defined in the USGS Quaternary fault map (USGS, 2020). The increase in basin depth reaches its maximum just south of downtown LA, as is seen in the southern part of Figure $11 \mathrm{~b}$ ) which shows the change in basin depth. The Upper Elysian Park fault is shown by a thick dashed cyan line in the center-right of the panels of Figure 11, and demarcates a steep gradient in the edge of the basin that has been accentuated as a result of the inversion. In Figure 12, this large jump in the depth of the basin edge occurs in the center of the profile A-A', with Figure 12 c) showing that the deep parts of the basin to the SSW of the fault are significantly slower in our final model, with the edge of the basin being significantly steeper in our model in a) than the reference model in b). This steepening is spatially coincident with the observations of high amplification further north in the data than in the reference models, seen in Figure 3, particularly in the 5-7 s band. Extracting the average basin edge gradient from 11.25-13.25 km along profile $\mathrm{A}-\mathrm{A}^{\prime}$ in Figure 12 gives a dip angle of $72-73^{\circ}$. The SCEC CVMs have evolved from the original models of Magistrale et al. (1996, 2000). For the Los Angeles basin, an empirically determined velocity law for compacted sediments is used (Faust, 1951). The velocity profiles are controlled by the depth of contacts between two large scale units (the Repettian and Mohnian), the inferred basement depth, and the age of the surface, as digitized from Yerkes et al. (1965) and Wright (1991). The results of Wright (1991)) rely on geological information from control wells. Wright's work in turn initialized the SCEC CVMs, either as a starting model for full-waveform inversion as used in CVM-S (Lee et al., 2014) or by acting as constraints in CVM-H (Tape et al., 2009; Shaw et al., 2015). There is a notable gap in the location of the control wells across the steep north- 
eastern boundary of the basin that is now covered by the CSN, leading to uncertainty about the basin geometry in prior works. Given the position of the basin sidewall is situated between the imbricated blind-thrust faults of the Elysian Park system (Plesch et al., 2007), the high apparent dip angle imaged by surface-wave measurements gives further support to an overthrusted basin in this region (as is included in the CVM-H model, albiet further to the northeast than is suggested by our results). Further cross-sections through the model are shown in Figure 14, and show that this steep basin sidewall continues along the northwest-southeast axis of the northern LA basin wall.

The second notable finding is that the depth of the low velocity zone in the hilly terrain north of the Los Angeles basin is substantially shallower than in the reference model, which can be seen both along the northern edge of Figure 11 and in the faster velocities around end A' of the transect in Figure $12 \mathrm{c}$ ). This shallowing of the basin relative to the CVM-S model is to be expected given the high Love wave speeds recorded in the northeast of the array from eikonal tomography, and the relatively lower amplification when compared to the slow, deep sediments in the central basin. Indeed, the northeastern components of the CSN operate within the surface expression of the lower Puente and Topanga units of the LA basin stratigraphic column, which were assembled early within the LA basin sequence and support a shallow sequence of basin rocks towards to the right of profile A-A' (Yerkes et al., 2005). In the Supplement, we further discuss these two main features in the context of fitting the rule-based CVM1 (Magistrale et al., 1996, 2000) to profile A-A'. By perturbing the locations of the loosely constrained geological contacts that define the CVM1, we analyse the outcomes of our fully 3D inversion in terms of geological structure, and find that the steep basin sidewall is consistent with recently $(\leq 4 \mathrm{Ma})$ active deformation.

\section{Conclusion}

We use Love waves generated by the Mw 6.4 and Mw 7.1 Ridgecrest, CA earthquakes to obtain Love-wave phase velocities and relative amplitudes between 4-10 s period using the Caltech-LAUSD Community Seismic Network, which offers unprecedented high-density coverage of the northeast LA basin. We use the level-set method of Muir \& Tsai (2020) to develop a parsimonious velocity inversion that updates the SCEC CVM-S background model only where empirical estimates of data uncertainty indicate additional complexity is warranted. By employing fully 3D surface-wave inversion, we avoid internal artifacts in the model and make best use of a relatively small dataset. In doing so, we find that the northeast wall of the LA basin is substantially steeper than that of the CVM-S model, allowing for high amplifications of surface waves in the $4-6$ s period band travelling within the basin. The constraints provided by this model cover some of the parts of LA with the highest density of population, infrastructure and commercial development, and highlight the continued importance of seismic veloc- 
ity model evolution in providing the most accurate possible estimates of potential strong ground motions in this important city.

\section{Acknowledgments}

The authors would like to thank Rob Graves (USGS) for providing synthetic seismograms for the Ridgecrest events. This study was supported by the United States National Science Foundation awards EAR-1520081, EAR-2105358 and EAR-2011079, and the Southern California Earthquake Center award 20024. JBM acknowledges the support of the General Sir John Monash Foundation and the Origin Energy Foundation for support during his graduate studies. The CSN data used in this paper are freely available from http:// csn.caltech.edu/data. The TEKS inversion code may be found at https:// doi.org/10.5281/zenodo.5834927 (Muir, 2022). Data analysis codes can be found at https://doi.org/10.5281/zenodo.5823526 (Muir et al., 2022).

\section{Appendix A Hierarchical Ensemble Kalman Sampler}

The Ensemble Kalman Inversion (EKI) scheme was introduced by Iglesias et al. (2013) by deriving a state-variable augmented Ensemble Kalman Filter (Evensen, 1994, 2003) with dynamics that approximated the LevenbergMarquardt method. EKI acts as an efficient black-box optimizer for large scale PDE constrained problems for which it is intractable or infeasible to obtain gradients, and has been used successfully in practical geophysical applications (e.g. Muir \& Tsai (2020); Tso et al. (2021)). Subsequent to its initial formulation, much analysis on the EKI scheme has been performed by studying it as a continuous time gradient flow (Kovachki \& Stuart, 2018), rather than in its original formulation as a discrete time dynamical system. This has lead to the development of the Ensemble Kalman Sampler (EKS, Garbuno-Inigo et al. (2020)), an algorithm for approximate sampling of the posterior distributions of large-scale Bayesian PDE constrained inverse problems. We utilize a hierarchical variant of the EKS scheme in this study to sample the posterior distribution of our local model update — we will briefly reintroduce the EKS scheme as described in Garbuno-Inigo et al. (2020) and then outline our variant hierarchical formulation. In general, the objective of these schemes is to approximate a posterior distribution whose negative $\log$-posterior is of the form

$$
\Phi(u, d)=\|d-G(u)\|_{\Gamma}+R(u),
$$

where $\Gamma$ is the data noise covariance matrix, and where the regularization term $R(u)$ introduces prior information. For instance, a typical choice would be a Tikhonov style regularization term $R(u)=\|u\|_{C_{0}}$ for some prior covariance matrix $C_{0}$. The norms here are defined by $\|u\|_{A}=\langle u, u\rangle_{A}=u^{T} A^{-1} u$.

The EKS scheme is an ensemble-based approximation of a preconditioned overdamped Langevin equation, which is a stochastic differential equation (SDE) of the form

$$
\dot{u}=-C(u) \nabla_{u} \Phi(u)+\sqrt{2 C(u)} \dot{W}
$$


with $C(u)$ a preconditioning operator that depends on $u$ and $\dot{W}$ a Brownian motion term. It can be shown that the long-term behavior of this SDE gives rise to a trajectory that has a distribution given by $p(u \mid d) \propto \exp (-\Phi(u, d))$ - i.e. the desired target posterior (Gelman et al., 1997). In the EKS scheme, an ensemble of particles $U=\left\{u^{(j)}\right\}_{j=1}^{J}$ are used to approximate the gradient of the likelihood, and $C(u)$ is chosen to be the empirical covariance $C(U)=\frac{1}{J} \sum_{j=1}^{J}\left(u^{(j)}-\bar{u}\right)\left(u^{(j)}-\bar{u}\right)^{T}$, where overbars denote means across the particle ensemble. Preconditioning by the empirical covariance acts to approximate the local curvature of the posterior by the ensemble, giving accelerated convergence compared to the unconditioned equation in a similar manner to the difference between Newton's method and gradient descent. The dynamics of this system of particles are given by the following SDE (without the gradient approximation and for Tikhonov-style Gaussian priors)

$\dot{u}^{(j)}=\frac{1}{J} \sum_{k=1}^{J}\left\langle\left(\nabla_{u} G\left(u^{(j)}\right)\left(u^{(k)}-\bar{u}\right), G\left(u^{(j)}-d\right)\right\rangle_{\Gamma} u^{(k)}-C(U) C_{0}^{-1} u^{(j)}+\sqrt{2 C(U)} \dot{W}^{(j)}\right.$.

Making the ensemble approximation for the gradient of the forward operator $G$ allows us to rewrite this in a form without an explicit derivative:

$\dot{u}^{(j)}=\frac{1}{J} \sum_{k=1}^{J}\left\langle\left(G\left(u^{(k)}\right)-\bar{G}, G\left(u^{(j)}\right)-d\right)\right\rangle_{\Gamma} u^{(k)}-C(U) C_{0}^{-1} u^{(j)}+\sqrt{2 C(U)} \dot{W}^{(j)}$,

which is the equation solved by the EKS as described by Garbuno-Inigo et al. (2020). We will define $D(U)=\frac{1}{J} \sum_{k=1}^{J}\left\langle\left(G\left(u^{(k)}\right)-\bar{G}, G\left(u^{(j)}\right)-d\right)\right\rangle_{\Gamma}$ for future convenience, so that the dynamics for the whole ensemble are given by

$$
\dot{U}=U D(U)^{T}-C(U) C_{0}^{-1} U+\sqrt{2 C(U)} \dot{W} .
$$

We note that at the equilibrium of the ensemble where $\dot{U} \sim 0$, these dynamics heuristically suggest a balance between a Newton-style update of the ensemble (using an empirical covariance matrix to approximate the inverse Hessian), which will converge to the maximum a posteriori point, and the generation of correlated Gaussian noise scaled to the original ensemble. The average behavior of the ensemble at equilibrium therefore results in sampling a local Gaussian approximation of the posterior. A video illustrating the evolution of the ensemble for a toy problem is available in the supplement.

In geophysical problems the scale of appropriate regularization (i.e., the choice of operator $C_{0}$ for Tikhonov regularized problems) is often unknown. As such, much recent effort has been devoted to the development of hierarchical methods for solving inverse problems, in which the prior itself is to some degree unknown and is controlled by some number of hyperparameters (see e.g. Malinverno \& Briggs (2004)). Additionally, for large-scale problems with Gaussian priors, it may be beneficial for efficient sampling to perform a coordinate transformation into diagonalized coordinates that remove the correlations in the prior between hyperparameters and the main parameters used in the inverse problem, as will be described below. These parametriza- 
tions are known as whitened, non-centered hierarchical parametrizations (Chada, 2018; Chada et al., 2018; Chen et al., 2019). The set of parameters is given by a collection of "regular" parameters $\xi$ and hyperparameters $\theta$. For zeromean Gaussian priors, the coordinate transformation is given by $u=L(\theta) \xi$ for a Cholesky factor $C_{0}(\theta)=L(\theta) L(\theta)^{T}$. With this transformation, the prior for the parameters $\xi$ is simply a Gaussian with identity covariance matrix. The Cholesky decomposition is an expensive operation of order $O\left(N(\xi)^{3}\right)$ where $N(\xi)$ is the number of main parameters. Lindgren et al. (2011) showed explicitly how to approximate the coordinate transformations used in this study by solving a stochastic partial differential equation (SPDE), which can be substantially more efficient. For certain choices of prior covariance, and by defining known boundary conditions on a rectangular volume encompassing the model parameters, there are known analytic solutions for the appropriate eigenfunctions $\phi_{i}(\theta)$ and eigenvalues $\nu_{i}(\theta)$ with which to solve the SPDE such that truncation of the series of eigenfunctions has the smallest total mean squared error; these eigenfunction-eigenvalue pairs form the Karhunen-Loève (KL) expansion (Dashti \& Stuart, 2013). Using the KL expansion, $L(\theta) \xi \sim \sqrt{\nu_{i}(\theta)} \phi_{i}(\theta) \xi_{i}$. Using these known analytic eigenfunctions and appropriately truncating the KL expansion to a reasonable number of eigenfunctions can drastically increase the speed of performing the coordinate transformation; for the commonly used Whittle-Matérn family of covariance functions in a rectangular domain, the transform (assuming Neumann boundary conditions) can be calculated using the inverse discrete cosine transform for even greater efficiency (Chen et al., 2019).

The hyperparameters $\theta$ may have arbitrary priors $\rho$, which are typically non-Gaussian but do not depend on $\xi$; consequently the dynamics of the system follow (for ensembles $\Xi=\left\{\xi^{(j)}\right\}_{j=1}^{J}, \Theta=\left\{\theta^{(j)}\right\}_{j=1}^{J}$ )

$$
\begin{aligned}
& \dot{\Xi}=\Xi D(L(\Theta) \Xi)^{T}-C(\Xi) \Xi+\sqrt{2 C(\Xi)} \dot{W} \\
& \dot{\Theta}=\Theta D(L(\Theta) \Xi)^{T}+C(\Theta) \nabla_{\theta} \log (\rho(\Theta))+\sqrt{2 C(\Theta)} \dot{W} .
\end{aligned}
$$

These dynamics derive from the original EKS by considering an augmented state vector $u=[\xi, \theta]^{T}$ and allowing arbitrary priors, noting that for a standard Normal prior $\log (\rho(x))=\left(-x^{2}-\log (2 \pi)\right) / 2$, so $\frac{\log (\rho(x))}{\partial x}=-x$. We have furthermore neglected the cross-covariance terms $\operatorname{Cov}(\Xi, \Theta)$ and assumed a block-diagonal form for the preconditioning matrix, allowing us to decouple the dynamics as above. In order to solve these equations, we use the same split-step implicit scheme as Garbuno-Inigo et al. (2020), which is given by

$$
\begin{aligned}
& \Xi_{k+1}^{*}=\Xi_{k}-\Delta t_{k} \Xi_{k} D\left(L\left(\Theta_{k}\right) \Xi_{k}\right)^{T}-\Delta t_{k} C\left(\Xi_{k}\right) \Xi_{k+1}^{*} \\
& \Theta_{k+1}^{*}=\Theta_{k}-\Delta t_{k} \Theta_{k} D\left(L\left(\Theta_{k}\right) \Xi_{k}\right)^{T}+\Delta t_{k} C\left(\Theta_{k}\right) \nabla_{\theta} \log \left(\rho\left(\Theta_{k+1}^{*}\right)\right) \\
& \Xi_{k+1}=\Xi_{k+1}^{*}+\sqrt{2 \Delta t_{k} C\left(\Xi_{k}\right)} W(\Xi)_{k} \\
& \Theta_{k+1}=\Theta_{k+1}^{*}+\sqrt{2 \Delta t_{k} C\left(\Theta_{k}\right)} W(\Theta)_{k},
\end{aligned}
$$

where $W(\Xi)_{k}$ and $W(\Theta)_{k}$ are matrices of standard random normals of the same shape as $\Xi$ and $\Theta$ respectively. The timestep $\Delta t_{k}$ is calculated adaptively following Kovachki \& Stuart (2018). Given a reference timestep $\Delta t_{0}$ 
we have $\Delta t_{k}=\Delta t_{0} /\left(\left\|D\left(L\left(\Theta_{k}\right) \Xi_{k}\right)\right\|+\delta\right)$ where the norm on $D$ is the Frobenius norm and $\delta$ is an arbitrary positive constant. Unlike in Garbuno-Inigo et al. (2020), the inclusion of arbitrary non-Gaussian priors for the hyperparameters $\theta$ means that the implicit update is no longer linear, but as the dimension of $\theta$ is usually small, the cost of performing this update using an iterative nonlinear solver is normally not overly burdensome. In practice we use forward-mode automatic differentiation for arbitrary priors $\rho$ and the L-BFGS method (Liu \& Nocedal, 1989) for solving the implicit update for $\Theta$.

\section{References}

Ajala, R., \& Persaud, P. (2021, October). Effect of Merging Multiscale Models on Seismic Wavefield Predictions Near the Southern San Andreas Fault. Journal of Geophysical Research: Solid Earth, 126(10), e2021JB021915.

Aster, R. C., Borchers, B., \& Thurber, C. H. (2018). Parameter estimation and inverse problems. Elsevier.

Bowden, D. C., \& Tsai, V. C. $\quad$ (2017, January). Earthquake ground motion amplification for surface waves: Ground Motions for Surface Waves. Geophysical Research Letters, 44 (1), 121-127. doi: 10.1002/2016GL071885

Bowden, D. C., Tsai, V. C., \& Lin, F. C. $\quad$ (2015, March). Site amplification, attenuation, and scattering from noise correlation amplitudes across a dense array in Long Beach, CA. Geophysical Research Letters, 42(5), 1360-1367. doi: 10.1002/2014GL062662

Bowden, D. C., Tsai, V. C., \& Lin, F.-C. $\quad$ (2017, December). Amplification and Attenuation Across USArray Using Ambient Noise Wavefront Tracking: USArray Noise Amplitudes. Journal of Geophysical Research: Solid Earth, 122(12), 10,086-10,101. doi: 10.1002/2017JB014804

Brissaud, Q., Bowden, D. C., \& Tsai, V. C. $\quad$ (2020, June). Extension of the Basin Rayleigh-Wave Amplification Theory to Include Basin-Edge Effects. Bulletin of the Seismological Society of America, 110(3), 1305-1322. doi: $10.1785 / 0120190161$

Brocher, T. A. (2005, December). Empirical relations between elastic wavespeeds and density in the earth's crust. Bulletin of the Seismological Society of America, 95(6), 2081-2092. doi: 10.1785/0120050077

Castellanos, J. C., Clayton, R. W., \& Juarez, A. $\quad$ (2020, May). Using a TimeBased Subarray Method to Extract and Invert Noise-Derived Body Waves at Long Beach, California. Journal of Geophysical Research: Solid Earth, 125(5). doi: 10.1029/2019JB018855

Chada, N. K. (2018, January). Analysis of Hierarchical Ensemble Kalman Inversion. arXiv:1801.00847 [math].

Chada, N. K., Iglesias, M. A., Roininen, L., \& Stuart, A. M. (2018, May). Parameterizations for ensemble Kalman inversion. Inverse Problems, 34 (5), 055009. doi: 10.1088/1361-6420/aab6d9

Chen, V., Dunlop, M. M., Papaspiliopoulos, O., \& Stuart, A. M. （2019, March). Dimension-Robust MCMC in Bayesian Inverse Problems. arXiv:1803.03344 [stat].

Clayton, R. W., Heaton, T., Chandy, M., Krause, A., Kohler, M., Bunn, J., ... Aivazis, M. (2012, January). Community Seismic Network. Annals of Geophysics, 54(6), 738-747. doi: 10.4401/ag-5269

Clayton, R. W., Kohler, M., Guy, R., Bunn, J., Heaton, T., \& Chandy, M. (2020, March). CSN-LAUSD Network: A Dense Accelerometer Network in Los Angeles Schools. $\quad$ Seismological Research Letters, 91(2A), 622-630. ～doi: 
Dashti, M., \& Stuart, A. M. (2013, February). The Bayesian Approach To Inverse Problems. arXiv:1302.6989 [math].

Datta, A. (2018, March). SWRT: A package for semi-analytical solutions of surface wave propagation, including mode conversion, across transversely aligned vertical discontinuities. Geoscientific Instrumentation, Methods and Data Systems, 7(1), 101-112. doi: 10.5194/gi-7-101-2018

Evensen, G. (1994). Sequential data assimilation with a nonlinear quasi-geostrophic model using Monte Carlo methods to forecast error statistics. Journal of Geophysical Research, 99 (C5), 10143. doi: 10.1029/94JC00572

Evensen, G. (2003, November). The Ensemble Kalman Filter: Theoretical formulation and practical implementation. Ocean Dynamics, 53(4), 343-367. doi: 10 .1007/s10236-003-0036-9

Faust, L. Y. (1951, April). Seismic velocity as a function of depth and geologic time. GEOPHYSICS, 16(2), 192-206. doi: 10.1190/1.1437658

Fichtner, A., van Herwaarden, D.-P., Afanasiev, M., Simutè, S., Krischer, L., Çubuk-Sabuncu, Y., ... Igel, H. (2018, May). The Collaborative Seismic Earth Model: Generation 1. Geophysical Research Letters, 45(9), 4007-4016. doi: 10.1029/2018GL077338

Filippitzis, F., Kohler, M. D., Heaton, T. H., Graves, R. W., Clayton, R. W., Guy, R. G., .. Chandy, K. M. (2021, April). Ground motions in urban Los Angeles from the 2019 Ridgecrest earthquake sequence. Earthquake Spectra, 875529302110039. doi: 10.1177/87552930211003916

Garbuno-Inigo, A., Hoffmann, F., Li, W., \& Stuart, A. M. (2020, January). Interacting Langevin Diffusions: Gradient Structure and Ensemble Kalman Sampler. SIAM Journal on Applied Dynamical Systems, 19(1), 412-441. doi: $10.1137 / 19 \mathrm{M} 1251655$

Gelman, A., Gilks, W. R., \& Roberts, G. O. (1997, February). Weak convergence and optimal scaling of random walk Metropolis algorithms. The Annals of Applied Probability, 7(1). doi: 10.1214/aoap/1034625254

Gibou, F., Fedkiw, R., \& Osher, S. （2018, January). A review of level-set methods and some recent applications. Journal of Computational Physics, 353, 82-109. doi: 10.1016/j.jcp.2017.10.006

Graves, R. W. (1996). Simulating Seismic Wave Propagation in 3D Elastic Media Using Staggered-Grid Finite Differences. Bulletin of the Seismological Society of America, 86(4), 1091-1106.

Graves, R. W., \& Pitarka, A. (2010, October). Broadband Ground-Motion Simulation Using a Hybrid Approach. Bulletin of the Seismological Society of America, 100(5A), 2095-2123. doi: 10.1785/0120100057

Haney, M. M., \& Tsai, V. C. (2015, November). Nonperturbational surface-wave inversion: A Dix-type relation for surface waves. GEOPHYSICS, 80(6), EN167EN177. doi: 10.1190/geo2014-0612.1

Haney, M. M., \& Tsai, V. C. (2017, May). Perturbational and nonperturbational inversion of Rayleigh-wave velocities. GEOPHYSICS, 82(3), F15-F28. doi: 10.1190/ geo2016-0397.1

Haney, M. M., \& Tsai, V. C. (2020, January). Perturbational and nonperturbational inversion of Love-wave velocities. GEOPHYSICS, 85(1), F19-F26. doi: 10.1190/ geo2018-0882.1

Iglesias, M. A., Law, K. J. H., \& Stuart, A. M. (2013, April). Ensemble Kalman methods for inverse problems. Inverse Problems, 29(4), 045001. doi: 10.1088/0266 $-5611 / 29 / 4 / 045001$

Ingersoll, R. V., \& Rumelhart, P. E. (1999). Three-stage evolution of the Los Angeles basin, southern California. Geology, 27(7), 593-596.

Jia, Z., \& Clayton, R. W. (2021, May). Determination of Near Surface Shear-Wave 
Velocities in the Central Los Angeles Basin With Dense Arrays. Journal of Geophysical Research: Solid Earth, 126(5). doi: 10.1029/2020JB021369

Kohler, M. D., Filippitzis, F., Heaton, T., Clayton, R. W., Guy, R., Bunn, J., \& Chandy, K. M. (2020, November). 2019 Ridgecrest Earthquake Reveals Areas of Los Angeles That Amplify Shaking of High-Rises. Seismological Research Letters, 91(6), 3370-3380. doi: 10.1785/0220200170

Kovachki, N. B., \& Stuart, A. M. (2018, August). Ensemble Kalman Inversion: A Derivative-Free Technique For Machine Learning Tasks. arXiv:1808.03620 [cs, math, stat].

Lai, V. H., Graves, R., Zhan, Z., Yu, C., \& Helmberger, D. (2019). Analyzing Shallow Basin Effects in Los Angeles Basin using 3D Simulations and Dense Array Analysis. In SCEC Annual Meeting (Vol. Contribution 9804).

Lai, V. H., Graves, R. W., Yu, C., Zhan, Z., \& Helmberger, D. V. (2020, October). Shallow Basin Structure and Attenuation Are Key to Predicting Long Shaking Duration in Los Angeles Basin. Journal of Geophysical Research: Solid Earth, 125 (10). doi: 10.1029/2020JB019663

Lee, E.-J., Chen, P., Jordan, T. H., Maechling, P. B., Denolle, M. A. M., \& Beroza, G. C. (2014). Full-3-D tomography for crustal structure in Southern California based on the scattering-integral and the adjoint-wavefield methods. Journal of Geophysical Research: Solid Earth, 119(8), 6421-6451. doi: 10.1002/2014JB011346

Lin, F.-C., Li, D., Clayton, R. W., \& Hollis, D. (2013, July). High-resolution 3D shallow crustal structure in Long Beach, California: Application of ambient noise tomography on a dense seismic array. GEOPHYSICS, 78(4), Q45-Q56. doi: 10.1190/geo2012-0453.1

Lin, F.-C., \& Ritzwoller, M. H. （2011, September). Helmholtz surface wave tomography for isotropic and azimuthally anisotropic structure: Helmholtz surface wave tomography. Geophysical Journal International, 186(3), 1104-1120. doi: 10.1111/j.1365-246X.2011.05070.x

Lin, F.-C., Ritzwoller, M. H., \& Snieder, R. ～(2009, June). Eikonal tomography: Surface wave tomography by phase front tracking across a regional broad-band seismic array. Geophysical Journal International, 177(3), 1091-1110. doi: 10.1111/j.1365-246X.2009.04105.x

Lin, F. C., Tsai, V. C., \& Schmandt, B. （2014, August). 3-D crustal structure of the western United States: Application of Rayleigh-wave ellipticity extracted from noise cross-correlations. Geophysical Journal International, 198(2), 656-670. doi: $10.1093 /$ gji/ggu160

Lindgren, F., Rue, H., \& Lindström, J. (2011, September). An explicit link between Gaussian fields and Gaussian Markov random fields: The stochastic partial differential equation approach: Link between Gaussian Fields and Gaussian Markov Random Fields. Journal of the Royal Statistical Society: Series B (Statistical Methodology), 73(4), 423-498. doi: 10.1111/j.1467-9868.2011.00777.x

Liu, D. C., \& Nocedal, J. (1989, August). On the limited memory BFGS method for large scale optimization. Mathematical Programming, 45(1-3), 503-528. doi: 10 $.1007 / \mathrm{BF} 01589116$

Lysmer, J. (1970). Lumped Mass Method for Rayleigh Waves. Bulletin of the Seismological Society of America, 60(1), 89-\&.

Magistrale, H., Day, Steven, Clayton, Robert W., \& Graves, Robert. (2000, December). The SCEC Southern California Reference Three-Dimensional Seismic Velocity Model Version 2. Bulletin of the Seismological Society of America, 90(6B), S65-S76. doi: 10.1785/0120000510

Magistrale, H., McLaughlin, K., \& Day, S. (1996, August). A geology-based 3D velocity model of the Los Angeles basin sediments. Bulletin of the Seismological Society of America, 86(4), 1161-1166. 
Malinverno, A., \& Briggs, V. A. (2004, July). Expanded uncertainty quantification in inverse problems: Hierarchical Bayes and empirical Bayes. Geophysics, 69(4), 1005-1016. doi: 10.1190/1.1778243

Muir, J. B. (2022, January). TikhonovEnsembleKalmanSampling.jl. Zenodo. doi: 10 $.5281 /$ zenodo. 5823526

Muir, J. B., Clayton, R. W., Tsai, V. C., \& Brissaud, Q. (2022, January). Parsimonious velocity inversion applied to the Los Angeles Basin, CA. Zenodo. doi: 10 $.5281 /$ zenodo. 5823526

Muir, J. B., \& Tsai, V. C. (2020, February). Geometric and level set tomography using ensemble Kalman inversion. Geophysical Journal International, 220(2), 967980. doi: $10.1093 /$ gji/ggz472

Osher, S., \& Sethian, J. A. (1988, November). Fronts propagating with curvaturedependent speed: Algorithms based on Hamilton-Jacobi formulations. Journal of Computational Physics, 79(1), 12-49. doi: 10.1016/0021-9991(88)90002-2

Pitarka, A., Graves, R. W., \& Rodgers, A. J. (2019, December). Strong Ground Motion Simulation for the M7.1, 2019 Ridgecrest California Earthquake. AGU Fall Meeting Abstracts, 31 .

Plesch, A., Shaw, J. H., Benson, C., Bryant, W. A., Carena, S., Cooke, M., ... Yeats, R. (2007, December). Community Fault Model (CFM) for Southern California. Bulletin of the Seismological Society of America, 97(6), 1793-1802. doi: $10.1785 / 0120050211$

Qiu, H., Lin, F.-C., \& Ben-Zion, Y. （2019, September). Eikonal Tomography of the Southern California Plate Boundary Region. Journal of Geophysical Research: Solid Earth, 124(9), 9755-9779. doi: 10.1029/2019JB017806

Rasmussen, C. E., \& Williams, C. K. I. (2006). Gaussian processes for machine learning. Cambridge, Mass: MIT Press.

SciPy 1.0 Contributors, Virtanen, P., Gommers, R., Oliphant, T. E., Haberland, M., Reddy, T., ... van Mulbregt, P. (2020, March). SciPy 1.0: Fundamental algorithms for scientific computing in Python. Nature Methods, 17(3), 261-272. doi: 10.1038/s41592-019-0686-2

Shaw, J. H., Plesch, A., Tape, C., Suess, M. P., Jordan, T. H., Ely, G., .. Munster, J. (2015, April). Unified Structural Representation of the southern California crust and upper mantle. Earth and Planetary Science Letters, 415, 1-15. doi: 10.1016/j.epsl.2015.01.016

Süss, M. P., \& Shaw, J. H. (2003). Pwave seismic velocity structure derived from sonic logs and industry reflection data in the Los Angeles basin, California. Journal of Geophysical Research: Solid Earth, 108(B3). doi: 10.1029/2001jb001628

Taborda, R., Azizzadeh-Roodpish, S., Khoshnevis, N., \& Cheng, K. ～(2016, June). Evaluation of the southern California seismic velocity models through simulation of recorded events. Geophysical Journal International, 205(3), 1342-1364. doi: $10.1093 /$ gji/ggw085

Tape, C., Liu, Q., Maggi, A., \& Tromp, J. (2009, August). Adjoint Tomography of the Southern California Crust. Science, 325(5943), 988-992. doi: 10.1126/science .1175298

Tsai, V. C., \& Atiganyanun, S. (2014, October). Green's Functions for Surface Waves in a Generic Velocity Structure. Bulletin of the Seismological Society of America, 104 (5), 2573-2578. doi: 10.1785/0120140121

Tso, C.-H. M., Iglesias, M., Wilkinson, P., Kuras, O., Chambers, J., \& Binley, A. (2021, February). Efficient multiscale imaging of subsurface resistivity with uncertainty quantification using ensemble Kalman inversion. Geophysical Journal International, 225(2), 887-905. doi: 10.1093/gji/ggab013

USGS. (2020). U.S. Geological Survey and California Geological Survey, Quaternary fault and fold database for the United States, https://www.usgs.gov/naturalhazards/earthquake-hazards/faults, Accessed April 12, 2021. USGS. 
Valentine, A. P., \& Sambridge, M. (2020a, March). Gaussian process models-I. A framework for probabilistic continuous inverse theory. Geophysical Journal International, 220 (3), 1632-1647. doi: 10.1093/gji/ggz520

Valentine, A. P., \& Sambridge, M. (2020b, March). Gaussian process models-II. Lessons for discrete inversion. Geophysical Journal International, 220(3), 16481656. doi: $10.1093 /$ gji/ggz521

Wright, T. L. (1991). Structural Geology and Tectonic Evolution of the Los Angeles Basin, California. In Active Margin Basins (pp. 35-134). American Association of Petroleum Geologists: AAPG Special Volumes.

Yerkes, R. F., Campbell, R. H., Alvarez, R. M., \& Bovard, K. R. (2005). Preliminary geologic map of the Los Angeles $30^{\prime} \times 60^{\prime}$ Quadrangle, southern California. US Geological Survey Open File Report, 1019, 1559-1573.

Yerkes, R. F., McCulloh, T. H., Schoellhamer, J. E., \& Vedder, J. G. (1965). Geology of the Los Angeles Basin, California; an introduction (Tech. Rep. No. 420-A). United States Geological Survey. 